\title{
Gravity Recovery Using COSMIC GPS Data: Application of Orbital Perturbation Theory
}

by

Cheinway Hwang

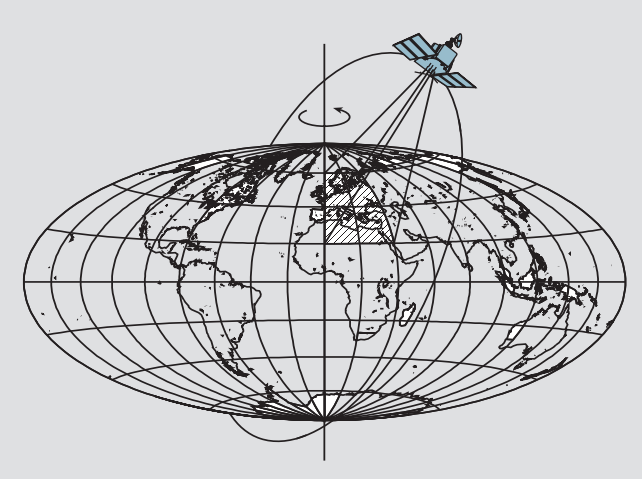

Report No. 463

Geodetic and GeoInformation Science

Department of Civil and Environmental Engineering and Geodetic Science The Ohio State University

Columbus, Ohio 43210-1275

October 1998 
Gravity recovery using COSMIC GPS data: application of orbital perturbation theory

\author{
by \\ Cheinway Hwang \\ Department of Civil Engineering, National Chiao Tung University, 1001 Ta Hsueh Road, \\ Hsinchu 300, Taiwan
}




\begin{abstract}
COSMIC is a joint Taiwan-US mission to study atmosphere using GPS occultation. Its GPS data for precise orbit determination can be used for gravity recovery. In this report a kinematic approach was employed which assumes the positional data can be derived from the GPS data of COSMIC in the operational phase. Using the geometric relationship between the positional variations of orbit and the variations in the six Keplerian elements, improved formulae for the radial, along-track and cross-track perturbations were derived. Based on a comparison with true perturbations from numerical integrations, these formulae are more accurate than the commonly used order-zero formulae. The improved formulae were used to simulate gravity recovery using the COSMIC data. In one simulation with the OSU91A model to degree 50 as the a priori geopotential model, it is demonstrated that the EGM96 model can be improved up to degree 26 using one year of COSMIC data.
\end{abstract}

A significant effort was devoted to the recovery of temporal gravity variation using COSMIC data. Sea level anomaly (SLA) was first generated using the Cycle 196 TOPEX/POSEIDON altimeter data. The steric anomaly due to thermal expansion was created using temperature data at 14 oceanic layers. The steric anomaly-corrected SLA was used to generate harmonic coefficients of temporal gravity variation. With a $3-\mathrm{cm}$ noise at a one-minute sampling interval in the COSMIC data, the gravity variation cannot be perfectly reproduced, but the recovered field clearly shows the gravity signature due to mass movement in an El Niño. With a $0.1-\mathrm{cm}$ noise, the temporal gravity variation up to harmonic degree 10 is almost exactly recovered and this prompts the need of a better processing technique and a sophisticated GPS receiver technology. 


\section{Acknowledgments}

The idea described in this report was initiated during my visit to the Ohio State University in the summer 1998, hosted by Prof. C.K. Shum. I am very grateful for his support and inspiring discussions. This research was partly supported by the National Science Council of ROC. Thanks also go to Dr. G. Balmino for providing me the program of inclination and eccentricity functions, and to Mr. S. -A. Chen for helping me to compute the steric anomaly. 


\section{Abstract}

\section{TABLE OF CONTENTS}

\section{Acknowledgments}

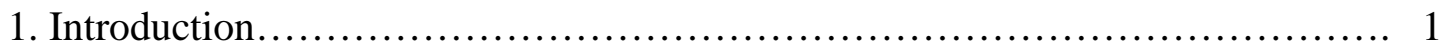

2. Methods and data type for gravity recovery from COSMIC $\ldots \ldots \ldots \ldots \ldots \ldots \ldots \ldots$

3. Orbital perturbations due to the geopotential............................. 6

3.1 Radial, along-track and cross-track perturbations................. 6

3.2 Higher order and resonance effects........................... 11

3.3 Model errors of the perturbation formulae....................... 12

4. Use of orbital perturbation formulae in gravity recovery .................... 17

5. Simulations of gravity recovery from COSMIC data...................... 18

5.1 Improving current gravity model............................. 18

5.2 Recovering temporal gravity variation.......................... 22

5.2.1 Generating gravity variation due to oceanic mass variation.........22

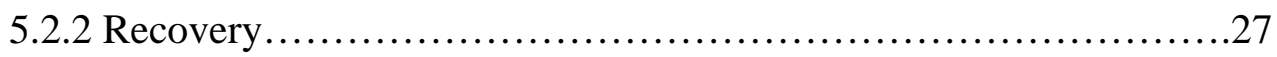

5.2.3 The effect of data noise on recovering temporal gravity.............30

6. Conclusion and recommendation...................................... 37

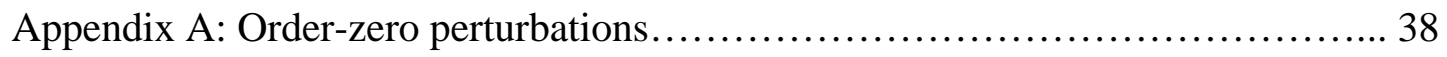

Appendix B: Computation of steric anomaly due to thermal expansion............40

Appendix C: Harmonic coefficients and geoid variation from corrected sea level anomaly................................ 41

Appendix D: Important FORTRAN programs................................43 
References.............................................................49

\section{TABLES}

Table 1: Orbital characteristics of COSMIC mission in the operational phase........2

Table 2: Model errors and statistics of the difference between the true and predicted perturbations............................................

Table 3: Statistics of sea level anomaly, steric anomaly and corrected sea level anomaly (in cm) over oceans with depth greater than $500 \mathrm{~m} . . . . . . . .$. 


\section{Figures}

Fig. 1: Three methods of gravity recovery from COSMIC GPS data and applications of gravity.

Fig. 2: Geometry showing the effects of the perturbations in argument of perigee (top), right ascension of the ascending node (center) and inclination (bottom) on the radial, along-track and cross-track perturbations of satellite position.......................................

Fig. 3: Differences between the true perturbation, and three predicted perturbations computed with three perturbation models (orderzero, $Q=1$ and $Q=2$ ). Day is the elapsed number of days since the first data point

Fig. 4: Difference between the true and predicted along-track perturbations using 10 and 15 coefficients in the empirical model. The perturbation model uses $Q=2$. Day is the elapsed number of days since the first data point

Fig. 5: Configuration of the COSMIC constellation in the operational phase at the initial epoch of orbit integration (0 hour UTC, Jan. 1, 2001). The 8 satellites are placed on 8 orbital planes with evenly spaced right ascensions and arguments of latitude

Fig. 6: Relative errors of the recovered harmonic coefficients from the degree-50 solution for (a) $\widehat{\bar{C}}_{n m}$ without constraint (b) $\widehat{\bar{S}}_{n m}$

without constraint (c) $\widehat{\bar{C}}_{n m}$ with constraint and (d) $\widehat{\bar{S}}_{n m}$ with constraint....20

Fig. 7: Relative errors of recovered zonal coefficients from the degree-50 
solution with constraint

Fig. 8: Geoid errors by degree computed from the coefficient errors of the EGM96 model, and the models from the COSMIC solutions using 7 days and 1 year of data.... 23

Fig. 9: TOPEX/POSEIDON-observed raw sea level anomaly (top), temperature-derived steric anomaly (center), and corrected sea level anomaly (by steric anomaly) at Cycle 196.

Fig. 10: Degree amplitude and cumulative percentage power of geoid variation computed from the corrected sea level anomaly in Fig. 9

Fig. 11: Contour maps of geoid variation up to degrees 5 (top), 15 (center) and 50 (bottom) from the corrected sea level anomaly in Fig. 9. Unit is $\mathrm{mm}$ .28

Fig. 12: True and modeled degree variances of gravity variation due to oceanic mass variation

Fig. 13: Perturbations of COSMIC orbit due to the mass variation of corrected sea level anomaly up to degree 50. Day is the elapsed number of days since the first data point.

Fig. 14: Relative errors of the recovered harmonic coefficients of gravity variation for (a) $\hat{\bar{J}}_{n w}$ and (b) $\widehat{\bar{K}}_{n m}$ using one week of COSMIC data and degree-50 solution 32

Fig. 15: Contour maps of recovered geoid variation up to degrees 5 (top), 15 (center) and 50 (bottom) using one week of 
COSMIC data and degree-50 solution. Unit is $\mathrm{mm}$.

Fig. 16: Relative errors of the recovered harmonic coefficients

of gravity variation using one week of COSMIC data and degree-15 solutions for (a) $\hat{\bar{J}}_{n m}$ (3-cm noise)
(b) $\widehat{\bar{K}}_{n m}$ (3-cm noise) (c) $\hat{\bar{J}}_{n m}$ (1-cm noise)
(d) $\widehat{\bar{K}}_{n m}$ (1-cm noise) (e) $\widehat{\bar{J}}_{n w}$ (0.1-cm noise)
(f) $\widehat{\bar{K}}_{n m}(0.1-\mathrm{cm}$ noise $)$.

Fig. 17: Contour maps of recovered geoid variation up to degrees 5 (top), 10 (center) and 15 (bottom) using one week of COSMIC data and degree-15 solution

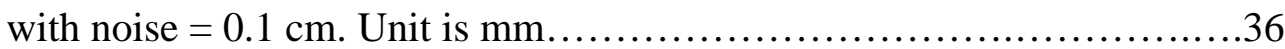




\section{Introduction}

The Constellation Observing System for Meteorology, Ionosphere, and Climate (COSMIC) is a joint Taiwan (ROC)-US satellite mission to study atmosphere using the GPS occultation technique. This mission is to be launched in 2003 and will deploy a constellation of 8 microsatellites, each equipped with one GPS receiver and two antennas. One antenna is to receive occulted signals for atmospheric study, and the other to receive un-occulted signals for precise orbit determination (POD) (Kuo and Lee, 1999). Although the COSMIC mission is primarily for atmospheric research, its POD GPS data can be used for geodetic research. In the early, geodetic phase of COSMIC, selected satellites will fly in the tandem mode at altitudes ranging from 300 $\mathrm{km}$ to $700 \mathrm{~km}$. When using the GPS data in the tandem mode the effect of the non-conservative forces can be reduced because the non-conservative forces acting on a satellite pair are highly correlated. In a simulation study Chao et al. (2000) show that the COSMIC GPS data from the geodetic phase can improve the accuracy of the EGM96 model (Lemoine et al., 1998) for harmonic coefficients up to degree 40 and for selected terms of higher degrees and orders.

The geodetic phase of COSMIC will last for less than one year and the mission will be soon shifted to the operational phase. Table 1 lists orbital characteristics of COMIC satellites in the operational phase, which are largely based on Kuo and Lee (1999). The 80 degree inclination of COSMIC is not officially determined, but any inclination less than 80 degree will introduce significant polar gaps in the data coverage and will not be ideal for global gravity recovery. In comparison, the GRACE and GOCE missions have polar and nearly polar obits that are ideal for global gravity recovery (Balmino et al., 1998). At the altitude of $800 \mathrm{~km}$, the gravity content sensible to the COSMIC satellites will drop to about harmonic degree of 50 (Hwang and Lin, 1998), so the data from the operational phase cannot produce high-frequency gravity information. Unlike the study of Chao et al. (2000), in this study we will use the COSMIC GPS data from the operational phase for gravity recovery. Since the operational phase has a lifetime of five years, it will be possible to see the time variation of gravity field with the COSMIC GPS data. The COSMIC orbit is nearly circular, thus, given the GPS-derived positional data of COSMIC satellites, it is convenient to use the linear orbit perturbation theory of Kaula (1966) for gravity computation as in the satellite altimetry research; see, e.g., Engelis (1987) and Hwang (1995). Kaula's theory is for the general case; for this study, perturbations in the radial, alongtrack and cross-track directions will be needed. Such perturbations can be derived from Kaula's theory, and formulae of various accuracies have been given by, e.g., Schrama (1991) and Rosborough and Tapley (1987). We will first derive alternative expressions for these perturbations with an aim to improve the efficiency and accuracy of the formulae in the linear theory. Then, the improved formulae will be validated and their accuracy will be assessed. Finally, these formulae will be used to compute the earth's gravity field from the COSMIC positional data in various simulations. The simulations will pay a special attention to recovering a time-varying gravity field due to oceanic mass movement derived from TOPEX/POSEIDON $(\mathrm{T} / \mathrm{P})$ altimeter data. 
Table 1: Orbital characteristics of COSMIC mission in the operational phase

\begin{tabular}{ll}
\hline Number of satellites & 8 \\
\hline Inclination & $80^{\circ}$ \\
Altitude & $800 \mathrm{~km}$ \\
Eccentricity & $\approx 0$ \\
orbital period & 101 minutes \\
Number of orbital planes & 8 \\
Nodal period & 314 days \\
Life time & 5 years \\
\hline
\end{tabular}




\section{Methods and data type for gravity recovery from COSMIC}

The primary purpose of this study is, by simulations, to recover the geopotential coefficients, $\bar{C}_{n m}$ and $\bar{S}_{n m}$, in the spherical harmonic representation of the earth's gravitational potential:

$V(r, \phi, \lambda)=\frac{G M}{r}\left[1+\sum_{n=2}^{\infty}\left(\frac{a_{e}}{r}\right)^{n} \sum_{m=0}^{n}\left(\bar{C}_{n m} \cos m \lambda+\bar{S}_{n m} \sin m \lambda\right) \bar{P}_{n m}(\sin \phi)\right]=\frac{G M}{r}+R$

where $R$ is the perturbing potential, GM is the product of Newton's gravitational constant and the earth's mass, $(r, \phi \lambda)$ are the spherical coordinates (radial distance, geocentric latitude, and longitude), $a_{e}$ is the semi-major axis of the earth's reference ellipsoid, $\bar{P}_{n m}$ is the fully normalized Legendre function of degree $n$ and order $m$ (Heiskanen and Moritz, 1985). The coordinate system is assumed to be geocentric, so the degree one terms are absent in (1). There are several methods for recovering the geopotential coefficients from the COSMIC GPS data. For example, Fig. 1 shows three possible methods of recovery, as well as applications of global gravity in engineering, oceanography, geophysics and other disciplines. In one method, called "dynamic method", we can treat carrier phases and psuedoranges of GPS just like regular satellite tracking data such as ranges from satellite laser ranging (SLR) and Doppler data from DORIS. Then, with a sophisticated software of orbit determination, e.g., NASA's GEODYN (Pavlis et al., 1996), we can solve for the geopotential coefficients, as well as the parameters of other perturbing forces and the initial state vectors. The dynamics of satellite are needed in this approach; see. e.g., Rim et al. (1996). Another method, called "kinematic method", is to use a GPS software to determine the precise positions of the COSMIC satellites without using satellite dynamics. The satellite position is a function of the perturbing forces acting on the satellite, including the force due to the geopotential. Using a linear orbital perturbation theory that links the satellite position to the geopotential, we can estimate the geopotential coefficients. In this kinematic method, the effect of the perturbing forces of non-gravity origin can be reduced by using a priori force models and further absorbed by an empirical model (see below). The third method in Fig. 1 first computes the GPS phase accelerations (Jekeli and Garcia, 1996), which are then used to compute satellite accelerations. Like satellite position, satellite acceleration is also a function of the geopotential, and in the rectangular coordinates the function is simply

$$
\left(\begin{array}{l}
a_{x} \\
a_{y} \\
a_{z}
\end{array}\right)=\nabla V=\left(\begin{array}{l}
\frac{\partial V}{\partial x} \\
\frac{\partial V}{\partial y} \\
\frac{\partial V}{\partial z}
\end{array}\right)
$$

where the acceleration components on the left side are computed from the GPS phases and the expressions of the gradient components of $V$ can be easily derived from (1) with a suitable coordinate transformation. Eq. (2) establishes the linear relationships between the observables 


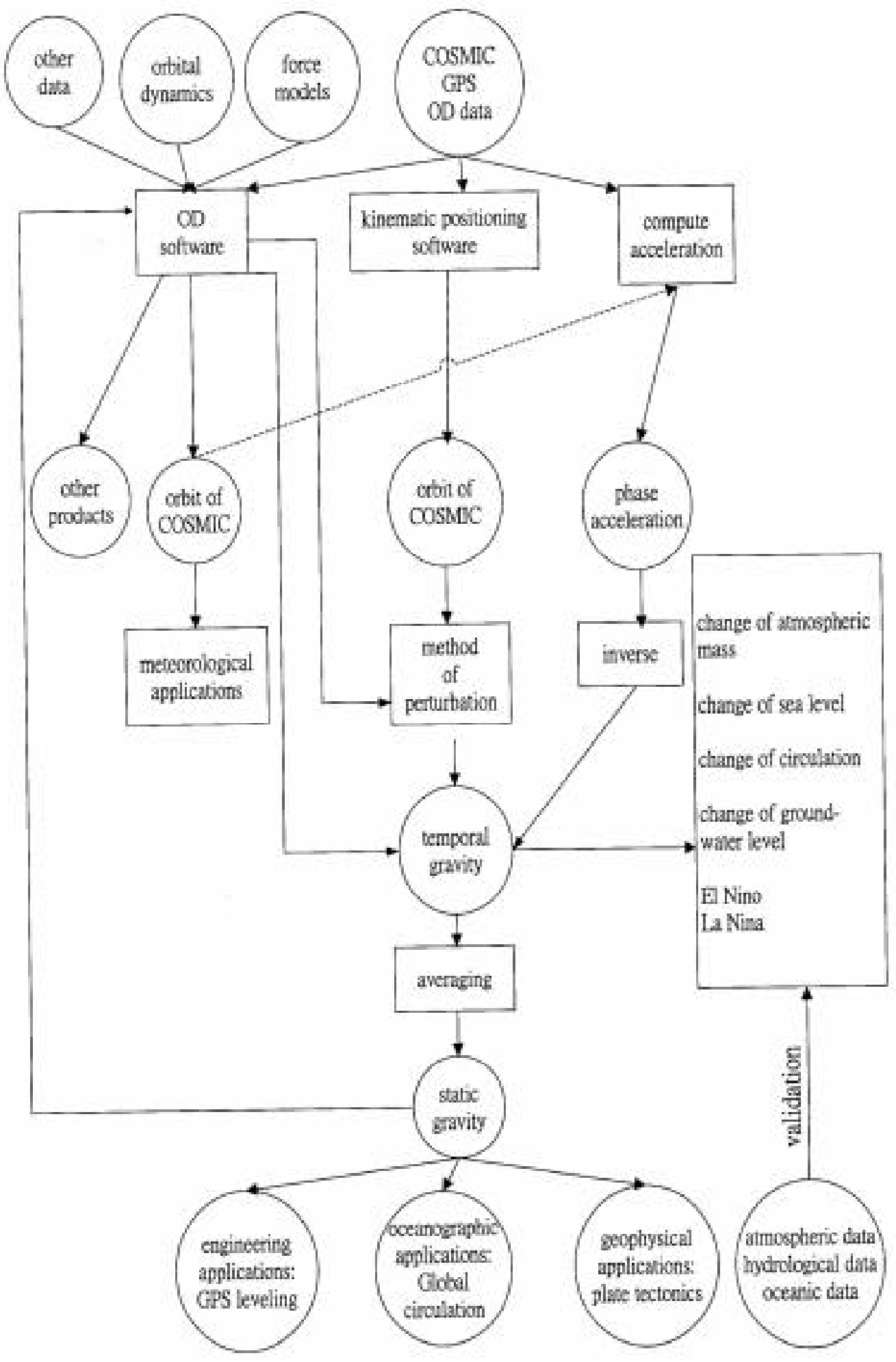

Fig. 1: Three methods of gravity recovery from COSMIC GPS data and applications of gravity. 
(i.e., the accelerations), and the parameters (i.e., the geopotential coefficients). However, the GPS-derived acceleration is again a combination of all perturbing forces, so a priori force models are needed to obtain the "pure" acceleration due to the geopotential.

Considering the limitation of our facility, in this study we will use the positional data of COSMIC to recover gravity, i.e., we will use the kinematic approach. The use of positional data of GPS-tracked satellite for gravity recovery has been recommended by, e.g., Schrama (1991) and Balmino et al. (1998). Like radial ranges from satellite altimetry, positional data of COSMIC can be used for gravity recovery, but there are two differences: (1) COSMIC positional data are three-dimensional, while altimeter ranges are one-dimensional, and (2) gravity recovery with altimeter ranges is affected by the dynamic oceanic topography, while COSMIC data are free from this effect. Furthermore, the GPS POD data of COSMIC will be sampled at $1 \mathrm{HZ}$ (Kuo and Lee, 1999), which far exceeds the needed sampling rate at the $800-\mathrm{km}$ altitude. Thus the positional data of COSMIC can be re-sampled at a lower rate, e.g., 1-minute interval, that is comparable with the shortest wavelength of gravity signal that can be sensed by the COSMIC satellites. In the case of 1-minute normal points, the accuracy of the positions of COMIC can be well better than the 10-cm accuracy of the GPS-determined T/P orbit (Bertiger et al., 1994). For example, Schrama (1991) and Balmino et al. (1998) assumed a 3-cm standard deviation for the GPS-determined positions in their simulations for the mission GOCE. Surely, in the year 2003 the accuracy of GPS determined orbit will be further improved. 


\section{Orbital perturbations due to the geopotential}

\subsection{Radial, along-track and cross-track perturbations}

To use the positional data of COSMIC, we will need to know the linear relationship between the satellite position and the geopotential coefficients. Such a relationship can be established using Kaula's theory of linear perturbation in the six Keplerian elements, whose notations are as follows:

semi-major axis of osculating orbital ellipse: $a$ eccentricity of osculating orbital ellipse: $e$ inclination: $I$ argument of perigee: $\omega$ right ascension of the ascending node: $\Omega$ mean anomaly: $M$

Given the three-dimensional, positional data of COSMIC, the analytical expressions for positional perturbations in the radial, along-track and cross-track directions are needed and are derived below. First, the radial distance of satellite from the geocenter can be expressed as

$$
r=a(1-e \cos E)
$$

where $E$ is the eccentricity anomaly. The perturbation in the radial direction is then

$$
\Delta x_{1}=\frac{\partial r}{\partial a} \Delta a+\frac{\partial r}{\partial e} \Delta e+\frac{\partial r}{\partial E} \Delta E=(1-e \cos E) \Delta a-(a \cos E) \Delta e+(a e \sin E \Delta E)
$$

Furthermore, as shown in Fig.2, the perturbations in the along-track and cross-track directions can be expressed as

$$
\begin{aligned}
& \Delta x_{2}=r(\Delta u+\Delta \Omega \cos I)=r[\Delta \omega+\Delta f+(\cos I) \Delta \Omega \\
& \Delta x_{3}=r[(\sin u) \Delta I-(\sin I \cos u) \Delta \Omega
\end{aligned}
$$

where $f$ is true anomaly and $u=\omega+f$ is argument of latitude. The perturbations in (4), (5) and (6) are already expressed in terms of the perturbations in the Keplerian elements, except $\Delta f$ and $\Delta E$. Complicated expressions of $\Delta f$ and $\Delta E$ in terms of Keplerian perturbations using infinite series expansions can be found in Rosborough and Tapley (1987). Because the sole purpose of the perturbation formulae is to serve as the linear functions for computing the design matrix in estimating the geopotential coefficients (see below), it will be possible to use simplified, rigorous expressions of perturbations that are free from infinite series expansions. First, the following relationships hold: 

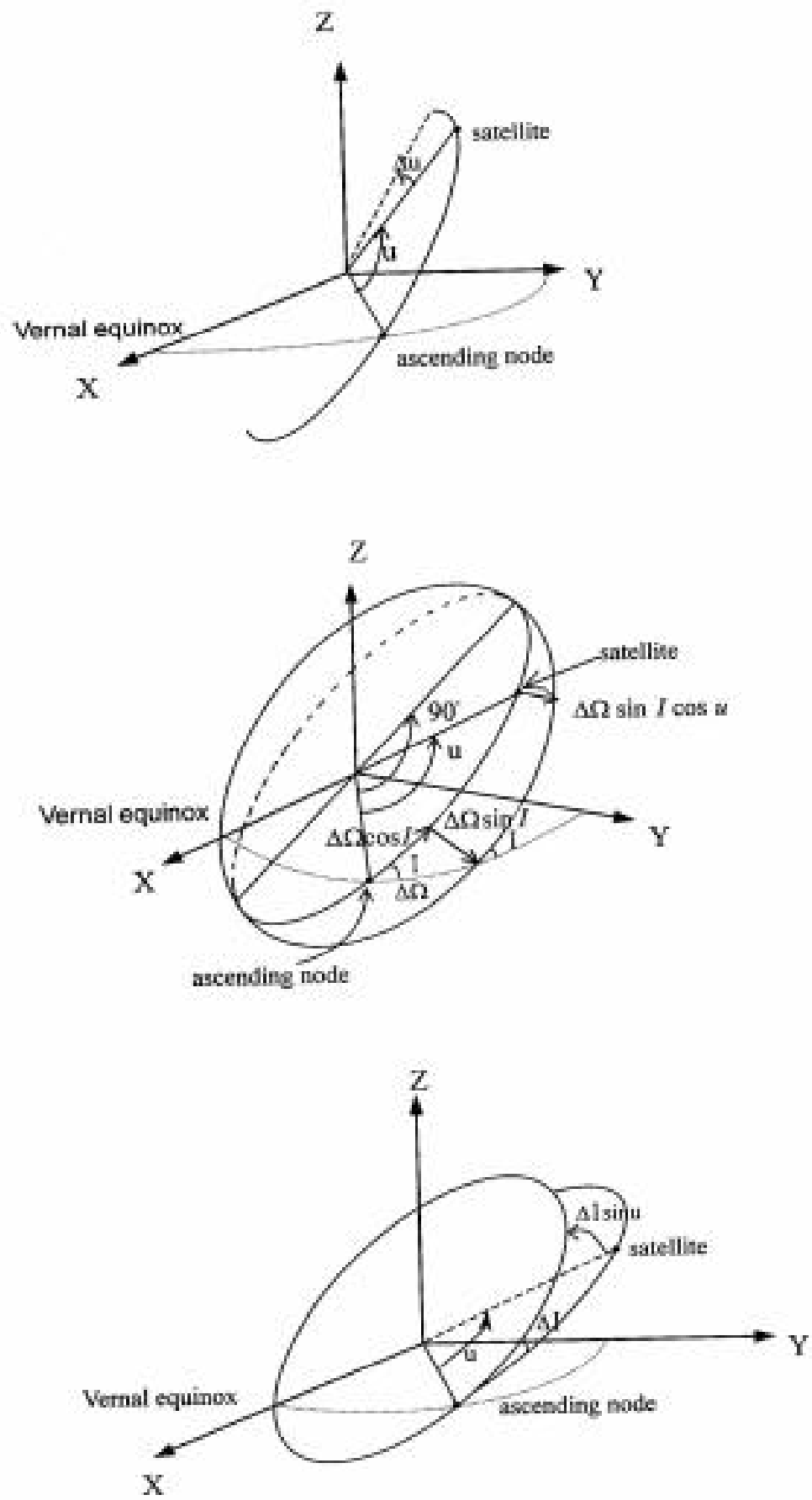

Fig. 2: Geometry showing the effects of the perturbations in argument of perigee (top), right ascension of the ascending node (center) and inclination (bottom) on the radial, along-track and cross-track perturbations of satellite position. 


$$
\begin{aligned}
& M=E-e \sin E \\
& \cos f=\frac{\cos E-e}{1-e \cos E}, \sin f=\frac{\sqrt{1-e^{2}} \sin E}{1-e \cos E}
\end{aligned}
$$

From (7), we have

$\Delta M=\frac{\partial M}{\partial E} \Delta E+\frac{\partial M}{\partial e} \Delta e=(1-e \cos E) \Delta E-(\sin E) \Delta e$

Re-arranging (9), wet get

$$
\Delta E=\frac{1}{1-e \cos E} \Delta M+\frac{\sin E}{1-e \cos E} \Delta
$$

Using a similar derivation, we have from (8)

$$
\begin{aligned}
\Delta f & =\frac{\sqrt{1-e^{2}}}{1-e \cos E} \Delta E+\frac{\sin E}{\sqrt{1-e^{2}}(1-e \cos E)} \Delta e \\
& =\frac{\sqrt{1-e^{2}}}{(1-e \cos E)^{2}} \Delta M+\frac{\left(2-e^{2}-e \cos E\right) \sin E}{\sqrt{1-e^{2}}(1-e \cos E)^{2}} \Delta e
\end{aligned}
$$

Let $\Delta_{k}, k=1, \cdots, 6$ be the perturbations of the six Keplerian elements in the order of $a, e, I, \Omega \omega$ and $M$. Substituting (10) and (11) into (4), (5) and (6), we have

$\Delta x_{i}=\sum_{k=1}^{6} c_{k}^{i} \Delta s_{k}$

where the coefficients are

$$
\begin{gathered}
c_{3}^{1}=c_{4}^{1}=c_{5}^{1}=0, c_{1}^{1}=1-e \cos E, c_{2}^{1}=-a \cos E+\frac{a e \sin ^{2} E}{1-e \cos E}, c_{6}^{1}=\frac{a e \sin E}{1-e \cos E} \\
c_{1}^{2}=c_{3}^{2}=0, c_{2}^{2}=\frac{r\left(2-e^{2}-e \cos E\right) \sin E}{\sqrt{1-e^{2}}(1-e \cos )^{2}}, c_{4}^{2}=r \cos I, c_{5}^{2}=r, c_{6}^{2}=\frac{r \sqrt{1-e^{2}}}{(1-e \cos E)^{2}} \\
c_{1}^{3}=c_{2}^{3}=c_{5}^{3}=c_{6}^{3}=0, c_{3}^{3}=\frac{r\left[\sin \omega(\cos E-e)+\sqrt{1-e^{2}} \cos \omega \sin E\right]}{1-e \cos E}, \\
c_{4}^{3}=r \sin I \frac{\left[\cos \omega(\cos E-e)-\sqrt{1-e^{2}} \sin \omega \sin E\right]}{1-e \cos E}
\end{gathered}
$$

In (13), $E$ is simply computed iteratively from $M$ and $e$ by (7) without using infinite series expansions, and $r$ is computed from $a, e$, and $E$ by (3). The perturbations in the Keplerian elements, $\Delta_{k}$, can be obtained by integrating Lagrange's equations of motion about a reference 
orbit that has fixed $a, e, I$ and linearly precessing $\Omega \omega M$ (Kaula, 1966; Balmino, 1994). For later development, the expressions of $\Delta_{k}$ 's are now briefly discussed. First, considering satellite altitude and orbital eccentricity, we can express the perturbing potential $R$ in (1) as a function of the Keplerian elements in a truncated series:

$$
R=\sum_{n=2}^{K} \sum_{m=0}^{n} \sum_{p=0}^{n} \sum_{q=-Q}^{Q} R_{n m p q}
$$

where $K$ is the maximum degree of the spherical harmonic expansion depending on the satellite altitude, and $Q$ is a number that depends on the orbital eccentricity. For example, at an altitude of $800 \mathrm{~km}, K$ can be 50 , and for a nearly circular orbit $Q$ can be 1 ; see also the discussion in Balmino (1994). Furthermore,

$$
R_{n m p q}=\frac{G M a_{e}^{n}}{a^{n+1}} \bar{F}_{n m p}(I) G_{n p q}(e) S_{n m p q}(\omega, M, \Omega, \theta)
$$

where

$$
\begin{aligned}
& S_{n m p q}(\omega, M, \Omega, \theta)=\left(\begin{array}{l}
\bar{C}_{n m}^{+} \\
-\bar{S}_{n m}^{-}
\end{array}\right) \cos [(n-2 p) \omega+(n-2 p+q) M+m(\Omega-\theta)] \\
& +\left(\begin{array}{l}
\bar{S}_{n m}^{+} \\
\bar{C}_{n m}^{-}
\end{array}\right) \sin [(n-2 p) \omega+(n-2 p+q) M+m(\Omega-\theta)]
\end{aligned}
$$

and $\theta$ is Greenwich sidereal time, $\bar{C}_{n m}^{+}$and $\bar{S}_{n m}^{+}$are fully normalized spherical harmonic coefficients (see (1)) when $(n-m)$ is even, and $\bar{C}_{n m}^{-}$and $\bar{S}_{n m}^{-}$are such coefficients when $(n-m)$ is odd. $\bar{F}_{n m r}$ is the fully normalized inclination function and $G_{n p q}(e)$ is the eccentricity function; see Kaula (1966) and Heiskanen and Moritz (1985). The normalizing factor for $\bar{F}_{n m \nu}$ is exactly the same as the normalizing factor for $\bar{P}_{n m}$. Use of $\bar{F}_{n m r}$ is compatible with the use of normalized geopotential coefficients in (1). Let

$$
\begin{aligned}
& S_{n m p q}^{*}(\omega, M, \Omega, \theta)=\left(\begin{array}{c}
\bar{C}_{n m}^{+} \\
-\bar{S}_{n m}^{-}
\end{array}\right) \sin [(n-2 p) \omega+(n-2 p+q) M+m(\Omega-\theta)] \\
& \quad-\left(\begin{array}{l}
\bar{S}_{n m}^{+} \\
\bar{C}_{n m}^{-}
\end{array}\right) \cos [(n-2 p) \omega+(n-2 p+q) M+m(\Omega-\theta)]
\end{aligned}
$$

Then the perturbations in $a, e, I$ are 
$\Delta s_{i}=\sum_{n=2}^{K} \sum_{m=0}^{n} \sum_{p=0}^{n} \sum_{q=-Q}^{Q} \alpha_{n m p q}^{i} S_{n m p q}$

and the perturbations in $\Omega \omega M$ are

$\Delta s_{i}=\sum_{n=2}^{K} \sum_{m=0}^{n} \sum_{p=0}^{n} \sum_{q=-Q}^{Q} \alpha_{n m p q}^{i} S_{n m p q}^{*}$

The coefficients $\mathrm{O}_{n m p ،}^{i}$ in the order of $a, e, I, \Omega \omega M$ are

$\alpha_{n m p q}^{1}=2 a b \bar{F}_{n m p} G_{n p d}(n-2 p+q)$

$\left.\mathrm{O}_{n m p q}^{2}=b \frac{\left(1-e^{2}\right)^{1 / 2}}{e} \bar{F}_{n m p} G_{n p} \phi_{q}\left(1-e^{2}\right)^{1 / 2}(n-2 p+q)-n+2 p\right]$

$\mathrm{O}_{n m p q}^{3}=b \bar{F}_{n m p} G_{n p q} \frac{[(n-2 p) \cos I-m]}{\sin I\left(1-e^{2}\right)^{1 / 2}}$

$\mathrm{O}_{n m p q}^{4}=b \frac{\bar{F}_{n m p}^{\prime} G_{n p q}}{\sin I\left(1-e^{2}\right)^{1 / 2}}$

$\alpha_{n m p q}^{5}=b\left[\frac{\left(1-e^{2}\right)^{1 / 2}}{e} \bar{F}_{n m p} \bar{G}_{n p q}^{\prime}-\frac{\cos I}{\sin I\left(1-e^{2}\right)^{1 / 2}} \bar{F}_{n m p}^{\prime} G_{n p q}\right]$

$\alpha_{n m p q}^{6}=b \bar{F}_{n m p}\left[2(n+1) G_{n p q^{-}} \frac{\left(1-e^{2}\right)^{1 / 2}}{e} G_{n p q^{-}}^{\prime} 3 G_{n p q}(n-2 p+q) \frac{\bar{n}}{\psi_{h m p q}}\right]$

where

$$
\begin{aligned}
& b=\frac{\bar{n}}{\psi h m p q}\left(\frac{a_{e}}{a}\right)^{n} \\
& \bar{n}=\sqrt{\frac{G M}{a^{3}}}
\end{aligned}
$$

$\psi_{h m p q}=(n-2 p) \dot{\boldsymbol{\omega}}+(n-2 p+q) \dot{M}+m(\dot{\boldsymbol{\Omega}}-\dot{\boldsymbol{\theta}})$

$\bar{F}_{n m p}^{\prime}=\frac{\partial \bar{F}_{n m p}}{\partial I}, G_{n p q}^{\prime}=\frac{\partial G_{n p q}}{\partial e}$

In (23), $\dot{\theta}$ is the speed of the Greenwich sidereal time, which is about the earth's mean rotational rate $\left(7.292115 \times 10^{-5} \mathrm{rad} \mathrm{s}^{-1}\right)$, and the processing rates $\dot{\omega}, \dot{M}$, and $\Omega$ are computed by 
$\dot{\Omega}=\frac{3 \bar{n} C_{20} a_{e}^{2}}{2\left(1-e^{2}\right)^{2} a^{2}} \cos ^{2} I$

$\dot{\omega}=\frac{3 \bar{n} C_{20} a_{e}^{2}}{4\left(1-e^{2}\right)^{2} a^{2}}\left(1-5 \cos ^{2} I\right)$

$\dot{M}=\bar{n}-\frac{3 \bar{n} C_{20} a_{e}^{2}}{4\left(1-e^{2}\right)^{3 / 2} a^{2}}\left(3 \cos ^{2} I-1\right)$

where $C_{2 \mathrm{C}}$ is the second degree zonal harmonic (about -0.00108263 ). The perturbation in mean anomaly, $\Delta M$, has taken into account the variation in $M$ due to the change in the mean motion arising from the perturbation of the semi-major axis (the third term in (20)); see also Kaula (1966, p. 49). Including this second-order effect will improve the accuracy of the along-track perturbation model.

In the practical computations, each of the three positional perturbations can be represented as the inner product of two vectors:

$$
\Delta x_{i}=\boldsymbol{A}_{i}^{T} \beta
$$

where $\beta$ is a vector containing the geopotential coefficients and $A_{i}$ is a vector obtained by combining (12), (18) and (19). For an orbital arc shorter than about one week, the $a, e$ and $I$ elements in $\boldsymbol{O}_{n m p}^{i}$ c can be regarded as constants, so we can compute $\boldsymbol{O}_{n m p}^{i}$, only once for all terms up to degree $K$ and index $Q$ (see (14)). For a nearly-circular orbit, approximate, analytical expressions of $\Delta x_{i}$ 's can be obtained; see, e.g., Schrama (1991) and Rosborough and Tapley (1987). In particular, for the radial perturbation the so called "order-zero" perturbation" (Rosborough and Tapley, 1987) is frequently used in satellite altimetry in connection with orbit refinement and gravity improvement; see, e.g., Wagner (1985), Engelis (1987) and Hwang (1995). The order-zero formulae of $\Delta_{x_{i}}$ are derived in Appendix A, and their accuracies will be compared with those of the rigorous formulae given in this section.

As a final note, in all computations below the inclination function is computed by a FORTRAN subroutine "FINCRS" and the eccentricity function by a subroutine "GKAULAF", both supplied by G. Balmino (private communication, 1999); a brief mention of these programs is given by Balmino (1994). In fact, we also use the summation formula given by Kaula (1966, p. 34) to compute the inclination function, and the result is identical to that computed by "FINCRS" up to at least harmonic degree 50. In addition, for the order-zero formulae in Appendix A, there is no need to compute the eccentricity function.

\subsection{Higher order and resonance effects}

The linear theory described above does not account for the higher order perturbations, and the theory breaks down in the case of resonance. A detailed derivation of higher order perturbations can be found in Kaula (1966). However, since the higher-order perturbations are not linear 
functions of the geopotential coefficients, they are of no use for the gravity computation in this study. Resonance occurs when the frequency $\psi_{h m p q}$ in (21) approaches zero. Depending on the closeness of $\psi_{h m p q}$ to zero, there are different degrees of resonance, i.e., shallow, deep and perfect resonances (Reigber, 1989). For COSMIC at the 800-km altitude, we find that, if

$$
\frac{\mid \psi_{h m p q}}{\dot{M}}<0.01
$$

then the coefficients in (20) become excessively large and the perturbations computed by (12) do not agree with those from the direct numerical method (see below). Thus, if the condition in (27) is met, we simply set $\mathbf{O}_{\text {nmp }}^{i}=0$. Fortunately, these two effects can be modeled by simple, empirical formulae. An empirical formula for the radial perturbation can be found in, e.g., Colombo (1984). In this study, we adopt the following empirical model to account for the higher order and resonance effects for COSMIC:

$$
\begin{aligned}
& \Delta x_{l}^{e}=a_{0}^{j}+a_{1}^{i} \cos u+a a_{2} \sin u+a_{3}^{j} \sin 2 u+a_{4}^{i} t \cos u+ \\
& a_{5}^{i} t \sin u+a_{b}^{i} t^{2} \cos u+a^{i} t^{2} \sin u+a_{8}^{i} t+a_{9}^{i} t^{2}
\end{aligned}
$$

where $t$ is the time elapsed since a reference epoch and $a_{k}^{i}$ are the coefficients for the $i$ th perturbation component. Note that such an empirical model can also absorb partly the error in the initial state vector and errors in the force models in the parameter estimation involving satellite dynamics. The choice in (28) is based on the results in Colombo (1984), Engelis (1987) and Hwang (1995), and most importantly based on the numerical tests carried out in this study.

\subsection{Model errors of the perturbation formulae}

To see how the perturbation formulae perform, one can replace the geopotential coefficients in the formulae by the differences between two sets of geopotential coefficients to compute the predicted perturbations along the trajectory of the satellite. In this way the predicted perturbations are in theory the differences between the two satellite trajectories resulting from the uses of two different geopotential models in the equations of motion of the satellite. Next, the "true" perturbations can be obtained by differencing the two trajectories computed by strict numerical interrogations of equations of motion. For each component the model error of the perturbation formula can be defined as

$$
M_{e}=\frac{\Delta D}{D}
$$

where $D$ is the RMS value of the true perturbation, and $\Delta D$ is the RMS value of the difference between the true and the predicted perturbations.

We choose the EMG96 and OSU91A geopotential coefficients to form the true and predicted perturbations (actually the trajectory differences). The numerical integrations were carried out using the DVDQ integrator (Krogh, 1974); see also Hwang and Lin (1998). Table 2 shows the model errors and the statistics of the differences between the true and predicted perturbations for 
three perturbation models: the order-zero model in Appendix A, and the perturbation models formulated in this study with $Q=1$ and $Q=2$. The arc length for all cases is 7 days. For each model error we also experiment with two different expansion degrees: $K=10$ and $K=50$. (see (14) for $Q$ and $K$ ). From Table 2, we find that for all formulae, the model error increases as $K$ increases. That is, the perturbation formulae are less accurate for a high degree expansion than for a low one. The largest model error is in the along-track component and the smallest model error is in the cross-track component. For the along-track component, the case with $Q=2$ improves the model accuracy only marginally compared to the case with $Q=1$. For all components, changing from $Q=1$ to $Q=2$ doubles the computing time. Also, the model with $\mathrm{Q}$ $=1$ outperforms the order-zero model and requires less computing time.

Fig. 3 shows the differences between the true perturbation and the predicted perturbations based on the three models, for the radial component and for $K=50$. The cm-level error in the

Table 2: Model errors and statistics of the difference between the true and predicted perturbations

(a) To harmonic degree 10, arc length $=7$ days

\begin{tabular}{lclcc}
\hline Type of model & $\begin{array}{l}\text { Model error }(\%) \\
(\mathrm{r} / \mathrm{a} / \mathrm{c})\end{array}$ & $\begin{array}{l}\text { Max. diff. }(\mathrm{cm}) \\
(\mathrm{r} / \mathrm{a} / \mathrm{c})\end{array}$ & $\begin{array}{l}\text { Min. diff. }(\mathrm{cm}) \\
(\mathrm{r} / \mathrm{a} / \mathrm{c})\end{array}$ & $\begin{array}{l}\text { RMS diff. }(\mathrm{cm}) \\
(\mathrm{r} / \mathrm{a} / \mathrm{c})\end{array}$ \\
\hline Order-zero model & $1.08 / 1.21 / 0.92$ & $1.1 / 6.1 / 0.8$ & $-1.5 /-4.6 /-1.3$ & $0.4 / 1.80 / 0.3$ \\
This study, $\mathrm{Q}=1$ & $0.85 / 0.64 / 0.49$ & $1.0 / 3.3 / 0.5$ & $-0.8 /-2.1 /-0.6$ & $0.3 / 0.9 / 0.2$ \\
This study, $\mathrm{Q}=2$ & $0.42 / 0.0 .53 / 0.49$ & $0.4 / 2.5 / 0.5$ & $-0.4 /-1.5 /-0.6$ & $0.1 / 0.8 / 0.2$ \\
\hline
\end{tabular}

(b) To harmonic degree 50, arc length $=7$ days

\begin{tabular}{lllll}
\hline Type of theory & $\begin{array}{l}\text { Model error }(\%) \\
(\mathrm{r} / \mathrm{a} / \mathrm{c})\end{array}$ & $\begin{array}{l}\text { Max. diff. }(\mathrm{cm}) \\
(\mathrm{r} / \mathrm{a} / \mathrm{c})\end{array}$ & $\begin{array}{l}\text { Min. diff. }(\mathrm{cm}) \\
(\mathrm{r} / \mathrm{a} / \mathrm{c})\end{array}$ & $\begin{array}{l}\text { RMS diff. }(\mathrm{cm}) \\
(\mathrm{r} / \mathrm{a} / \mathrm{c})\end{array}$ \\
\hline Order-zero theory & $1.52 / 3.43 / 3.38$ & $4.6 / 34.8 / 10.5$ & $-4.6 /-33.9 /-7.8$ & $1.3 / 10.3 / 2.7$ \\
This study, $\mathrm{Q}=1$ & $1.18 / 1.10 / 0.93$ & $3.3 / 14.5 / 2.5$ & $-4.1 /-12.4 /-2.7$ & $1.0 / 3.5 / 0.7$ \\
This study, $\mathrm{Q}=2$ & $0.96 / 1.08 / 0.93$ & $2.7 / 9.4 / 2.5$ & $-2.7 /-10.7 /-2.7$ & $0.8 / 3.4 / 0.7$ \\
\hline
\end{tabular}

Note: $\mathrm{r}=$ radial, $\mathrm{a}=$ along-track, $\mathrm{c}=$ cross-track, $Q=$ upper limit of index in eccentricity function

models with $\mathrm{Q}=1$ and 2 is well smaller than the positional error of COMIC from GPS. In the differences in the along-track direction, for all models there are distinct components at 0.02 cycle per revolution (cpr) and 2-cpr components of time-dependent amplitude, thus we use the following 15-coefficient empirical model for the radial component:

$$
\begin{aligned}
& \Delta x_{l}^{e}=a_{0}^{i}+a_{1}^{i} \cos u+a_{2}^{i} \sin u+a_{3}^{i} \sin 2 u+a_{4}^{i} t \cos u+a_{5}^{i} \sin u+a_{6}^{i} t^{2} \cos u+a_{7}^{i} t^{2} \sin u+a_{8}^{i} t \\
& +a_{9}^{i} t^{2}+a_{10}^{i} \cos 2 u+a_{11}^{i} t \cos 2 u+a_{12}^{i} t \sin 2 u+a_{13}^{i} \cos 0.02 u+a_{14}^{i} \sin 0.02 u
\end{aligned}
$$




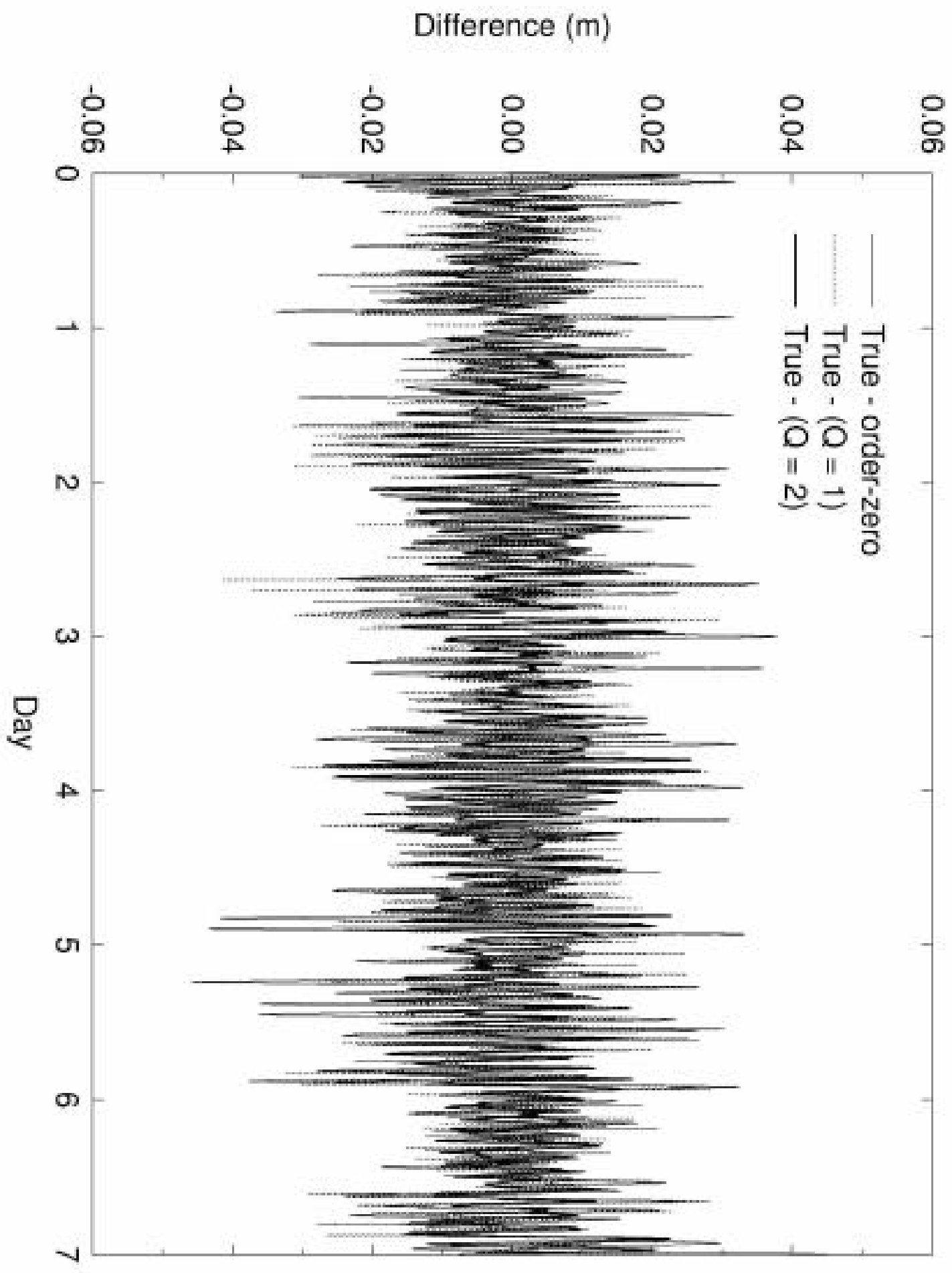

Fig. 3: Differences between the true perturbation, and three predicted perturbations computed with three perturbation models (order-zero, $Q=1$ and $Q=2$ ). Day is the elapsed number of days since the first data point. 
Fig. 4 shows the differences in the along-track direction resulting from the uses of the 10coefficient model (see (28)) and the 15-coefficient model (see (30)) in the model with Q $=2$. Clearly, the 10-coefficient model cannot account for the large difference near the end of the arc. Use of the 15-coefficient model reduces the RMS error from $8.3 \mathrm{~cm}$ to $3.4 \mathrm{~cm}$, which is comparable to the assumed positional error of COSMIC. Note that, when estimating geopotential coefficients from the positional data, we can use iterations to reduce the effect of model error, because the successively estimated coefficients will become stable and the perturbations will approach zero.

In summary, based on the model errors in Table 2 and the assumed data noise, we will use the perturbation model with $Q=1$ in all simulations below. The maximum arc length that is considered valid for the reference orbit in the perturbation model and the empirical model is 7 days; a longer arc should be divided into multiple segments of 7-day arc with each segment having a new reference orbit and a new set of empirical coefficients. 


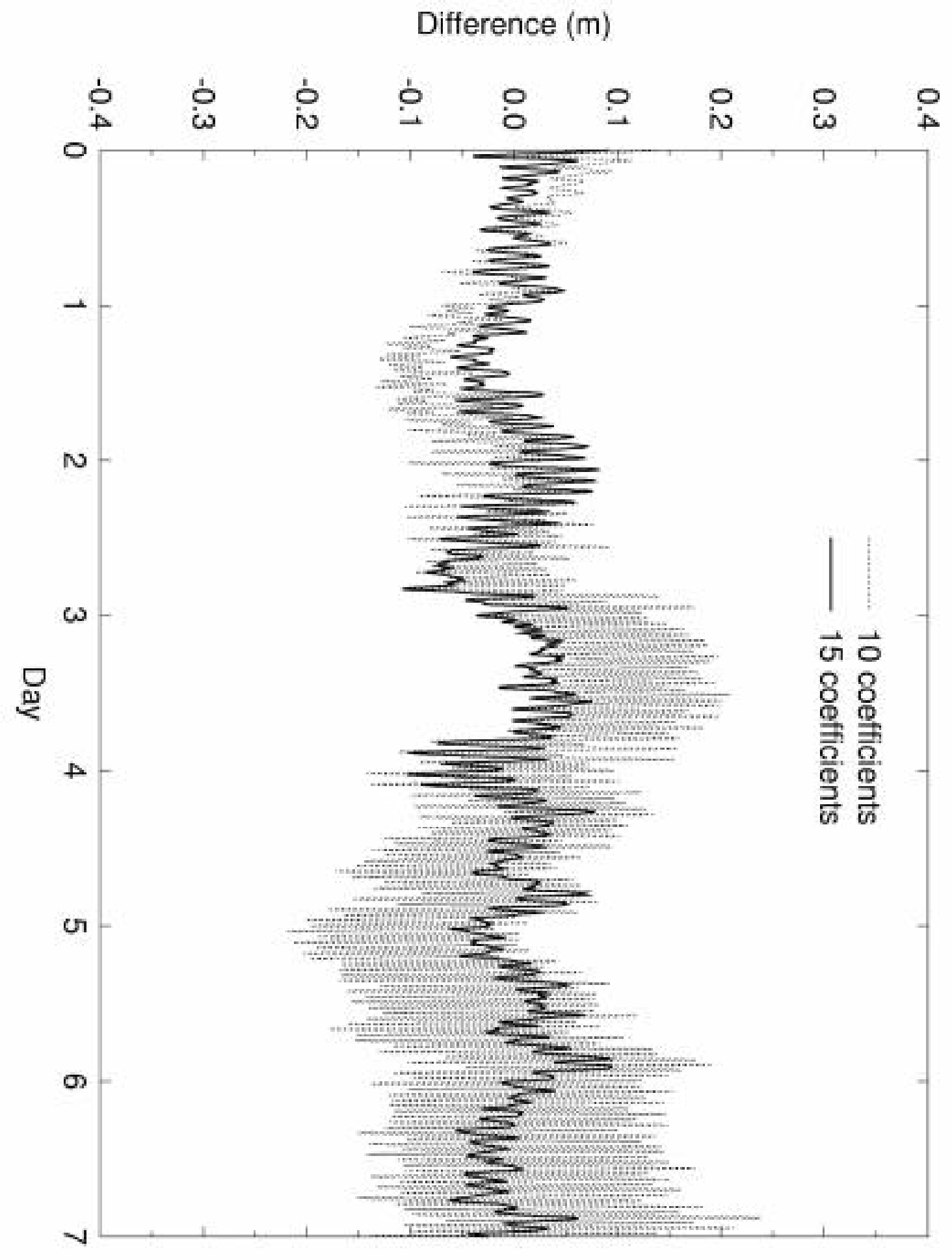

Fig. 4: Difference between the true and predicted along-track perturbations using 10 and 15 coefficients in the empirical model. The perturbation model uses $Q=2$. Day is the elapsed number of days since the first data point. 


\section{Use of orbital perturbation formulae in gravity recovery}

The linear perturbation formulae can be used to compute the design matrix when estimating the geopotential coefficients. Given the positional data of COSMIC, at each epoch we can set up observation equations in the radial, along-track and cross-track directions as

$$
x_{i}+v_{i}=x_{i}^{0}\left(\beta_{0}\right)+\left.\frac{\Delta x_{i}}{\beta}\right|_{\beta=\beta_{0}} \Delta \beta+\Delta x_{i}^{e}(\zeta), \quad i=1,2,3
$$

where $x_{i}$ 's are the positional data with noise $v_{i}$ 's, $\beta$ is a vector containing the geopotential coefficients, $\beta_{0}$ is an approximate of $\beta, \Delta \beta$ is the correction with $\beta=\beta_{0}+\Delta \beta$, $\zeta$ contains the empirical coefficients and finally $x_{i}^{0}$ is an approximate of $x_{i}$ that is computed with $\beta_{0}$ and other a priori force models. If the a priori force models are not perfect, but their errors are dominated by the long-wavelength components, then the empirical models in (28) and (30) will absorb most of the errors. Let

$$
x=\left[\begin{array}{c}
\Delta \beta \\
\zeta
\end{array}\right]
$$

Then $\boldsymbol{X}$ can solved by the least-squares method:

$\boldsymbol{X}=\left(\boldsymbol{A}^{T} \boldsymbol{P A}+\boldsymbol{P}_{X}\right)^{-1} \boldsymbol{A}^{T} \boldsymbol{P L}$

where $\boldsymbol{A}$ is the design matrix composed of $\frac{\partial \Delta x_{i}}{\partial \beta}$ and the function values of the empirical model, vector $\boldsymbol{L}$ contains the residual observations, i.e., $x_{i}-x_{i}^{0}, \boldsymbol{P}$ is the weight matrix of $\boldsymbol{L}$, and $\boldsymbol{P}_{\boldsymbol{x}}$ is the a priori weight matrix of $\boldsymbol{X}$. Assuming that the variances of the geopotential coefficients follow modified Kaula's rule (Reigber, 1989), i.e.,

$\bar{\sigma}_{n}^{2}=\frac{1}{2 n+1} \sum_{m=0}^{n}\left(\bar{C}_{n m}^{2}+\bar{S}_{n m}^{2}\right) \approx 5 \times 10^{-11} n^{-4}$

Then $P_{x}=\operatorname{diag}\left(p_{C_{n m}}, p S_{n m}\right)$ is an diagonal matrix with its elements computed by

$p C_{n m}=p S_{n m}=\frac{1}{\overline{\mathrm{\sigma}}_{n}^{2}}$

Use of $\boldsymbol{P}_{\boldsymbol{x}}$ is necessary for data of non-global coverage. More discussion on using weighted constraints in geopotential estimation from satellite tracking data can be found in, e.g., Reigber (1989). 


\section{Simulations of gravity recovery from COSMIC data}

\subsection{Improving current gravity model}

In this case we wish to see how current gravity models, especially the EGM96 model, can be improved using the positional data of COSMIC. The steps of simulation are as follows:

(1) Integrate a 7-day orbit at a 1-minute interval using the EGM96 coefficients for each of the 8 COSMIC satellites. Random errors are added to the orbits based on a standard deviation of $3 \mathrm{~cm}$ in GPS positioning. These orbits are treated as the positional data of COSMIC.

(2) Repeat (1), but with the OSU91A coefficients and without random errors. These orbits are treated as approximate orbits based on a priori geopotential coefficients (in this case, OSU91A)

(3) Subtract the orbits in (2) from the orbits in (1) to get the radial, along-track and cross-track perturbations.

(4) Estimate the differences between the EGM96 and OSU91A coefficients by least-squares using the linear perturbation theory with data from the "observed" perturbations in step (3).

At the initial epoch of integrations, the right ascensions of orbital planes and arguments of latitudes of the 8 satellites in the COSMIC constellation are shown in Fig. 5. The amount of data to process and the computing times in such a simulation are enormous. For example, the 87 -day arcs result in 8 normal matrices with a total size of 220 Megabytes, and forming one normal matrix requires $3 \mathrm{CPU}$ hours on a Pentium-III $600 \mathrm{MHZ}$ machine. The choice of 1- minute interval is based on the assumption that the shortest wavelength of the gravity field that COSMIC can sense corresponds to a degree-50 field (the actual maximum degree can be higher). At $800 \mathrm{~km}$, a COSMIC satellite will take about 6050 seconds to travel in one revolution. According to the sampling theorem (e.g., Meskó, 1994), the along-track sampling interval should be $6060 /(2 \times 50) \cong 60$ seconds. In general, the along-track sampling interval is

$$
\Delta=\frac{T}{2 K} \approx \frac{\pi a^{3 / 2}}{K \sqrt{G M}}
$$

where $T$ is the period of one revolution, $K$ is the maximum degree of the geopotential field (see (14)) used in the orbit integration, and $a$ is the semi-major axis of the mean orbital ellipse. However, to be compatible with the satellite cross-track spacing, the actual along-track sampling interval can be larger than that given in (35). For example, Chao et al. (2000) used a five-minute interval when deriving normal point data from the geodetic phase of COSMIC.

The recovered coefficients were then assessed by examining the external and internal accuracies. For the external accuracy we computed the relative error of a coefficient by

$$
E_{n m}^{c}=\left|\frac{\Delta \bar{C}_{n m}-\Delta \widehat{\bar{c}}_{n m}}{\Delta \bar{C}_{n m}}\right|, E_{n m}^{S}=\left|\frac{\Delta \bar{S}_{n m}-\Delta \widehat{\bar{S}}_{n m}}{\Delta \bar{S}_{n m}}\right|
$$

where $\Delta \widehat{\overline{\mathbf{C}}}_{\mathrm{nm}}$ and $\Delta \widehat{\overline{\mathrm{S}}}_{\mathrm{nm}}$ are the estimates of $\Delta \overline{\boldsymbol{C}}_{n m}$ and $\Delta \overline{\boldsymbol{S}}_{n m}$ (the differences between EGM96 and OSU91A coefficients). Fig.6 shows the relative errors of the recovered coefficients. For comparison, the relative errors of the recovered coefficients without weighted constraints are 


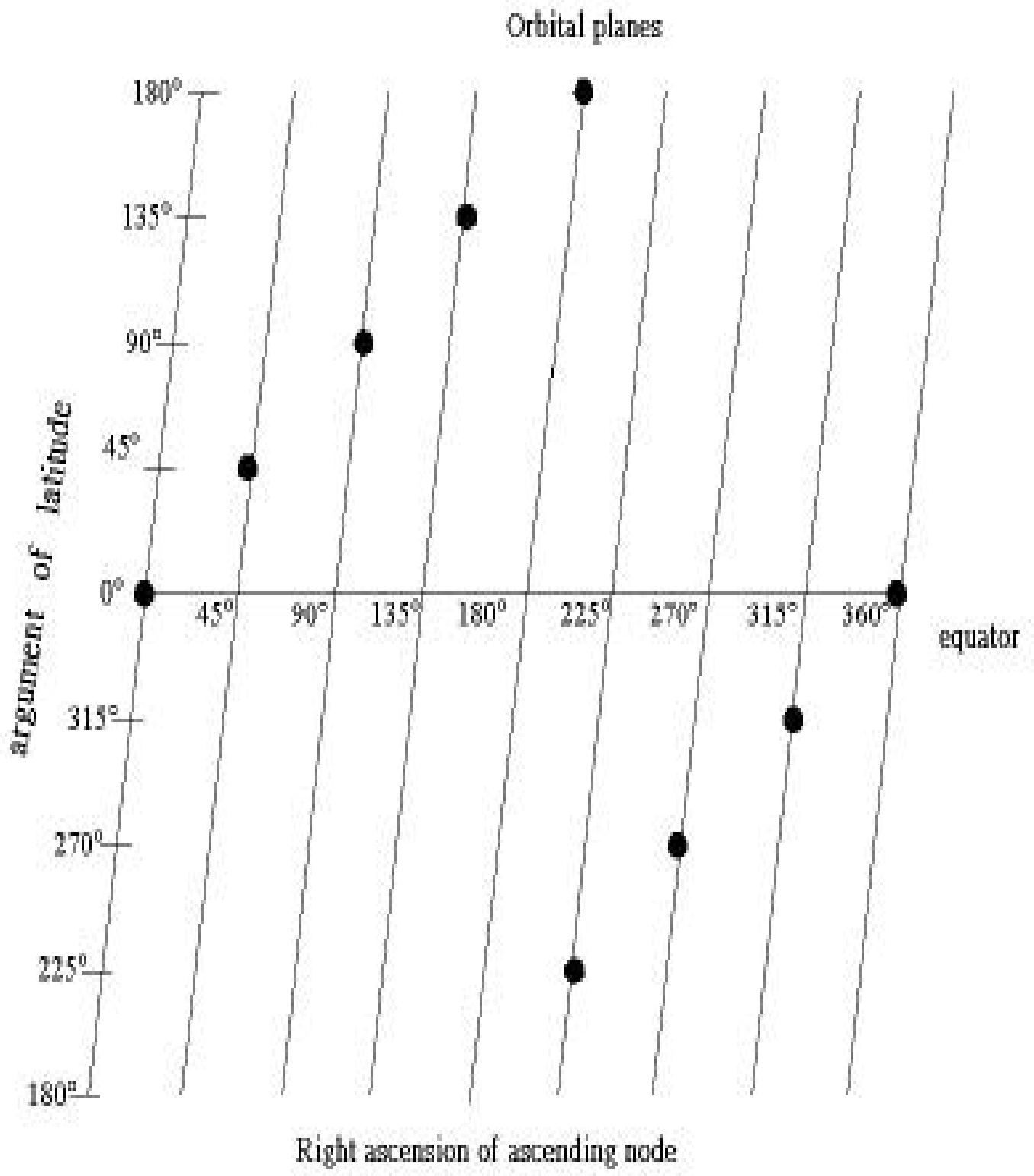

Fig. 5: Configuration of the COSMIC constellation in the operational phase at the initial epoch of orbit integration (0 hour UTC, Jan. 1, 2001). The 8 satellites are placed on 8 orbital planes with evenly spaced right ascensions and arguments of latitude. 

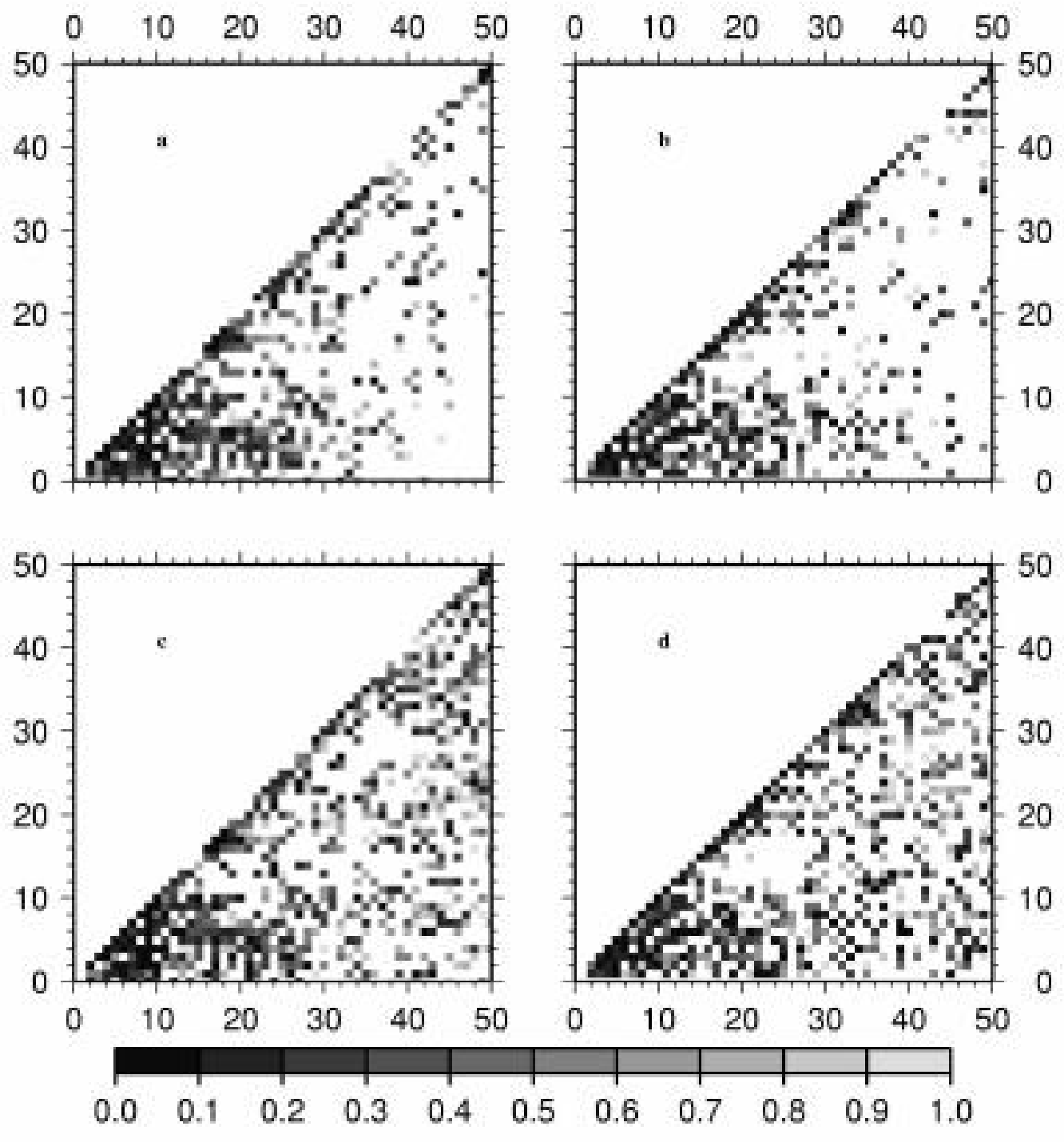

Fig. 6: Relative errors of the recovered harmonic coefficients from the degree-50 solution for (a) $\widehat{\bar{C}}_{n m}$ without constraint (b) $\widehat{\bar{S}}_{n m}$ without constraint (c) $\widehat{\bar{C}}_{n m}$ with constraint and (d) $\widehat{\bar{S}}_{n m}$ with constraint. 


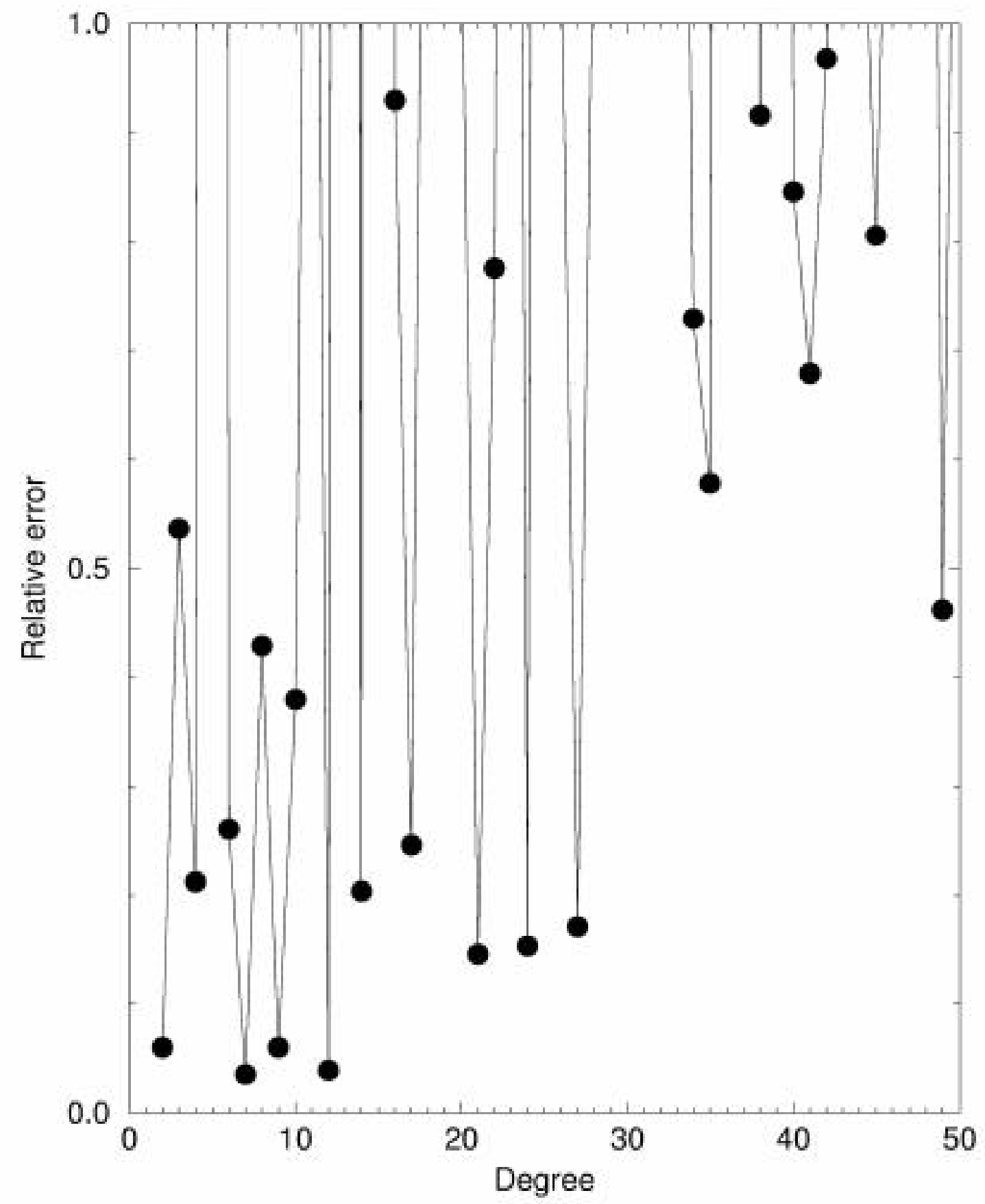

Fig. 7: Relative errors of recovered zonal coefficients from the degree-50 solution with constraint. 
also shown in Fig. 6. Indeed the constraints have reduced the relative errors considerably. For coefficients of degree lower than 10 , the relative errors are mostly below 0.1 . The relative errors of the sectorial coefficients are relatively small compared to the errors of other harmonics. Fig.7 shows the relative errors of the recovered zonal coefficients. In general, the odd zonal coefficients have relatively large errors, especially the $\bar{C}_{5,0}, \bar{C}_{15,0}$ and $\bar{C}_{37, \text { cterms. The }} \bar{C}_{2,0}$ term has an error of 0.05 , which is almost the smallest among all coefficients. The external errors will be further reduced if more than one week of COSMIC data are used.

Next we examined the internal accuracy using the geoid errors implied by the standard deviations of the recovered coefficients from the least-squares. Fig. 8 shows the geoid errors implied by the recovered field, and those implied by the EGM96 model. In Fig.8 we also show the geoid errors implied by the solution with 1 year of COSMIC data. As shown in Fig. 8, below degree 7 the geoid error from the one-week solution of COSMIC are comparable to that from EGM96, and beyond degree 7 the former is larger than the latter. However, for degrees below 26 the geoid error from the one-year solution is much smaller than that from EGM96. With five years of COSMIC data, improvement at degrees higher than 26 can be foreseen. On the other hand, improvement at higher degrees can be achieved by using the geodetic phase data that spans a relatively short time, since the gravity signal-to-noise ratio will be significantly magnified due to the lower altitude.

\subsection{Recovering temporal gravity variation 5.2.1 Generating gravity variation due to oceanic mass variation}

Next we wish to see the performance of COSMIC in recovering temporal gravity variation. The operational phase of COMIC may last as long as five years, so its data are ideal for determining temporal gravity variation, which is due to factors such as changes in oceanic mass, atmospheric mass, ground water level, ice-sheet thickness; see also Chao (1993) and Wahr et al. (1998) for useful discussions on this issue. In comparison to the earth's static gravity, the temporal gravity variation is very small and is further reduced at satellite altitude. In this simulation, we assume that the only source of gravity variation arises from the variation in the oceanic mass as observed by TOPEX/POSEIDON (T/P). T/P is a satellite altimeter mission specifically designed to measure sea surface heights (SSHs); see also Fu et al. (1994) for a complete description of this mission. As the parameters to be recovered, we generate spherical harmonic coefficients of gravity variation as follows:

(1) Average 5.6 years of T/P altimeter data to get mean SSHs.

(2) Compute the difference between the SSH of T/P Cycle 196 and the mean SSH. The difference is called sea level anomaly (SLA).

(3) Compute the steric anomaly in January 1994 (the month of T/P Cycle 196). ( see Appendix B)

(4) Subtract the steric anomaly from SLA, yielding corrected SLA (CSLA)

(5) Compute the spherical harmonic coefficients of the potential due to the mass of CSLA up to degree 180. (see Appendix C) 


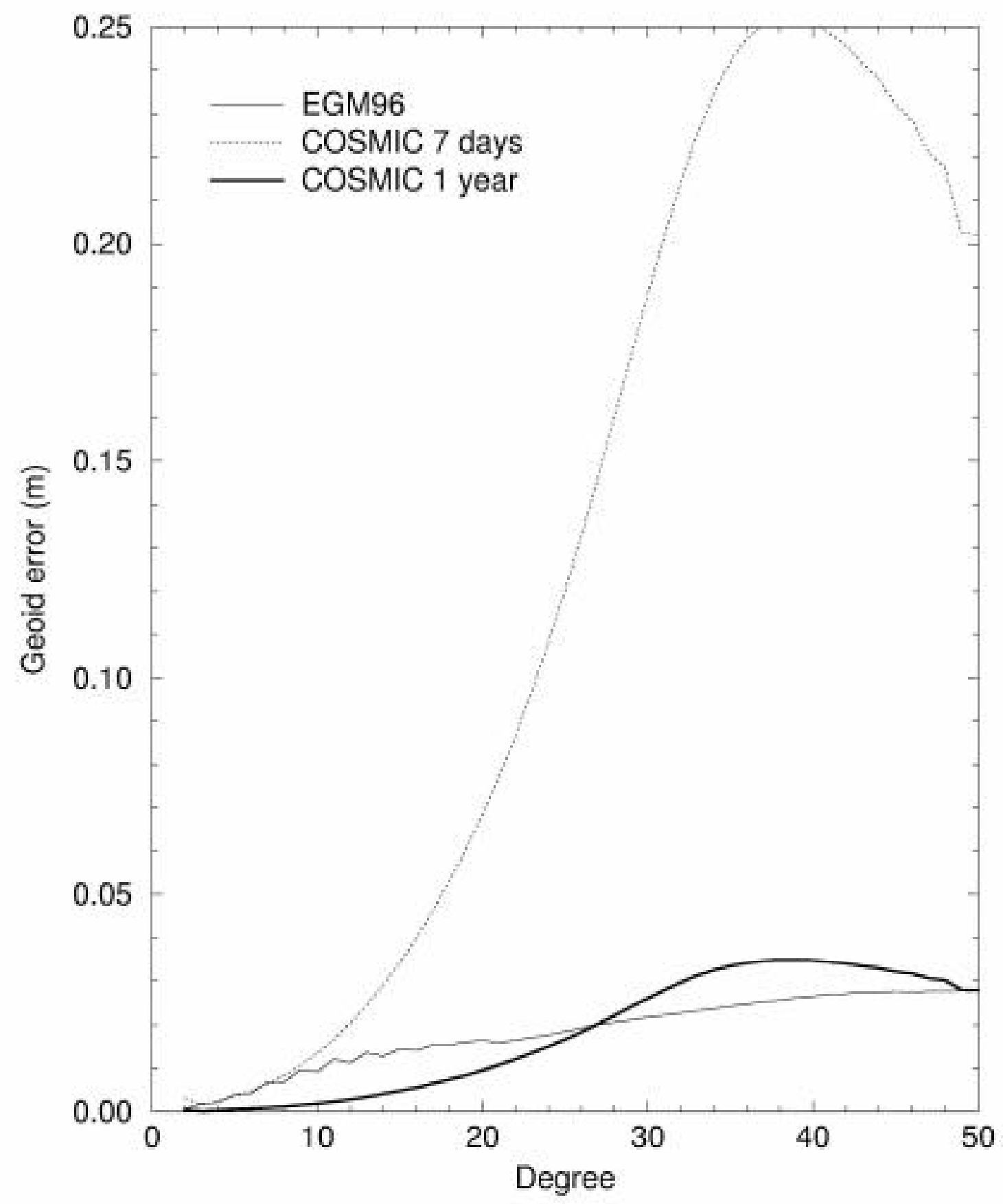

Fig. 8: Geoid errors by degree computed from the coefficient errors of the EGM96 model, and the models from the COSMIC solutions using 7 days and 1 year of data. 
The steric anomaly due to thermal expansion of the oceans will not cause mass variation, so it must be removed from the raw SLA in order to see the temporal, oceanic mass variation. Fig. 9 shows SLA and CSLA from T/P Cycle 196, and the steric anomaly, and Table 3 shows their statistics. Cycle 196 is selected to see the large variation of SSH over the Pacific Ocean and the Indian Ocean during the 1997-1998 El Niño. By comparing the RMS values of SLA and the steric anomaly in Table 3, we find that globally the steric anomaly contributes about half to the sea level variation. As seen in Fig. 9, the sea surface northeast of Australia dropped by as much as $20 \mathrm{~cm}$, while the sea surface in the central, eastern Pacific rose by about the same amount. Sea surface northeast of Madagascar also rose by more than $20 \mathrm{~cm}$. These lows and highs in the sea surface are the result of the 1997-1998 El Niño, and only partially due to the steric anomaly (one can compare the SLA and CSLA maps to see this). That is, during the

Table 3 Statistics of sea level anomaly, steric anomaly and corrected sea level anomaly (in $\mathrm{cm}$ ) over oceans with depth greater than $500 \mathrm{~m}$

\begin{tabular}{lllll}
\hline & Maximum & Minimum & Mean & RMS \\
\hline Sea level anomaly & 29.9 & -54.4 & 0.22 & 5.4 \\
Steric anomaly & 21.41 & -11.61 & 0.32 & 2.5 \\
Corr. sea level anomaly & 38.4 & -47.9 & -0.1 & 5.3 \\
\hline
\end{tabular}

1997-1998 El Niño, there were actual large mass variations northeast of Australia and in the central, eastern Pacific. Fig. 10 shows the degree amplitudes of the geoid variation due to the mass variation. Degree amplitude of geoid is defined as

$$
N_{n}=R_{e} \sqrt{\sum_{m=0}^{n}\left(\bar{J}_{n m}^{2}+\bar{K}_{n m}^{2}\right.}
$$

where $\bar{J}_{n m}$ and $\bar{K}_{n m}$ are the harmonic coefficients of the gravity variation, and $R_{\boldsymbol{e}}$ is the mean radius of the earth. For the CSLA-induced geoid variation, all degree amplitudes are smaller than $1 \mathrm{~mm}$, except at degrees 2 and 3 . Thus the geoid variation is indeed very small

compared to the total geoid (about 30 m RMS from EGM96 to degree 360). Also shown in Fig. 10 is the cumulative power up to degree $L$, defined as

$$
P_{L}=\frac{\sum_{n=2}^{L} N_{n}^{2}}{\sum_{n=2}^{N \max } N_{n}^{2}}
$$

where Nmax is the highest degree of expansion, which is 180 in this case. Based on Fig.10, the power of the geoid variation is concentrated at the low degree terms. For example, up to degrees $5,10,15,36$ and 50, the cumulative powers are 84.5\%, 96.8\%, 98.8\%, 99.8\%, and 99.9\%, respectively. Fig.11 shows the geoid variations expanded to degrees 5, 15 and 50. 


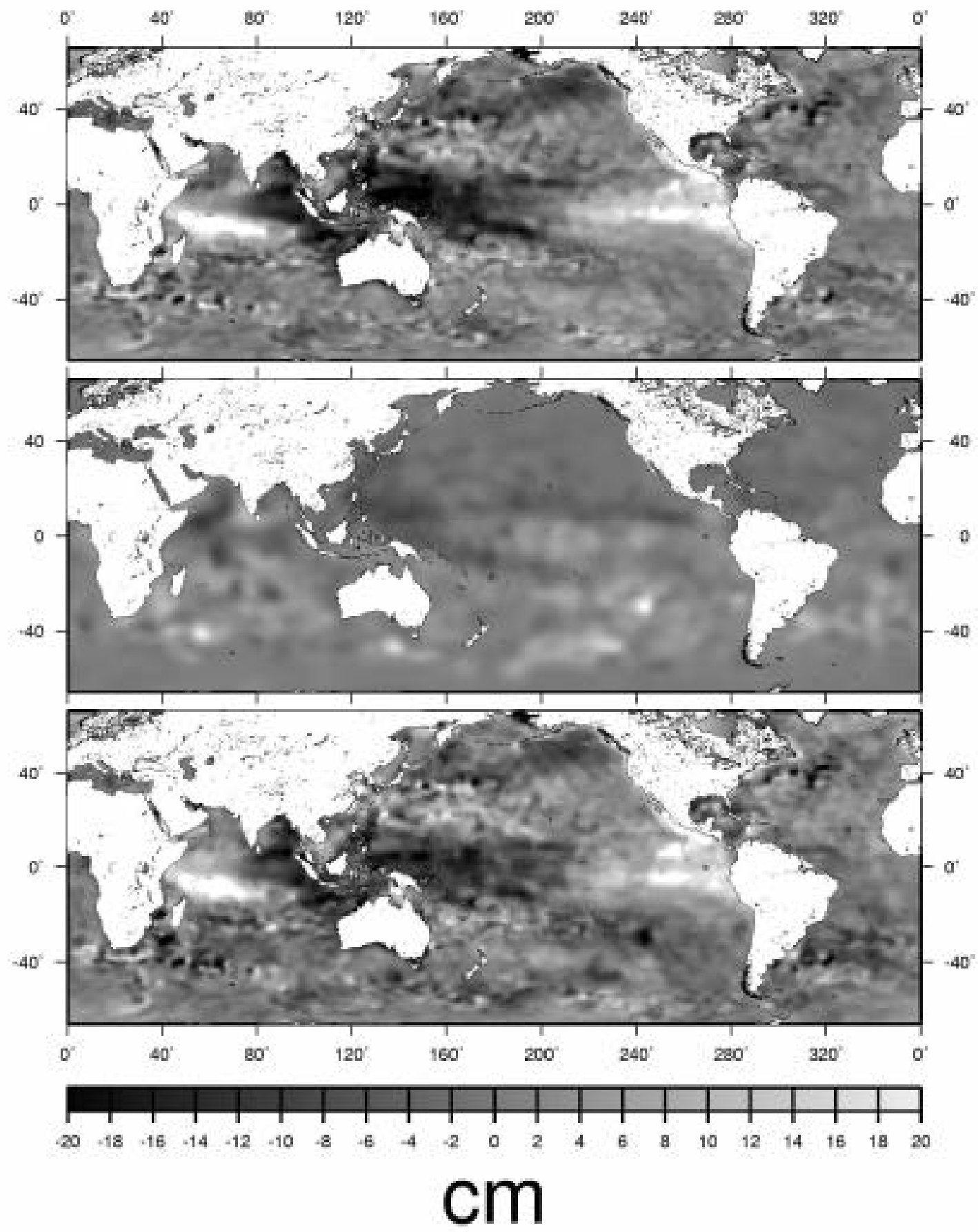

Fig. 9: TOPEX/POSEIDON-observed raw sea level anomaly (top), temperature-derived steric anomaly (center), and corrected sea level anomaly (by steric anomaly) at Cycle 196. 


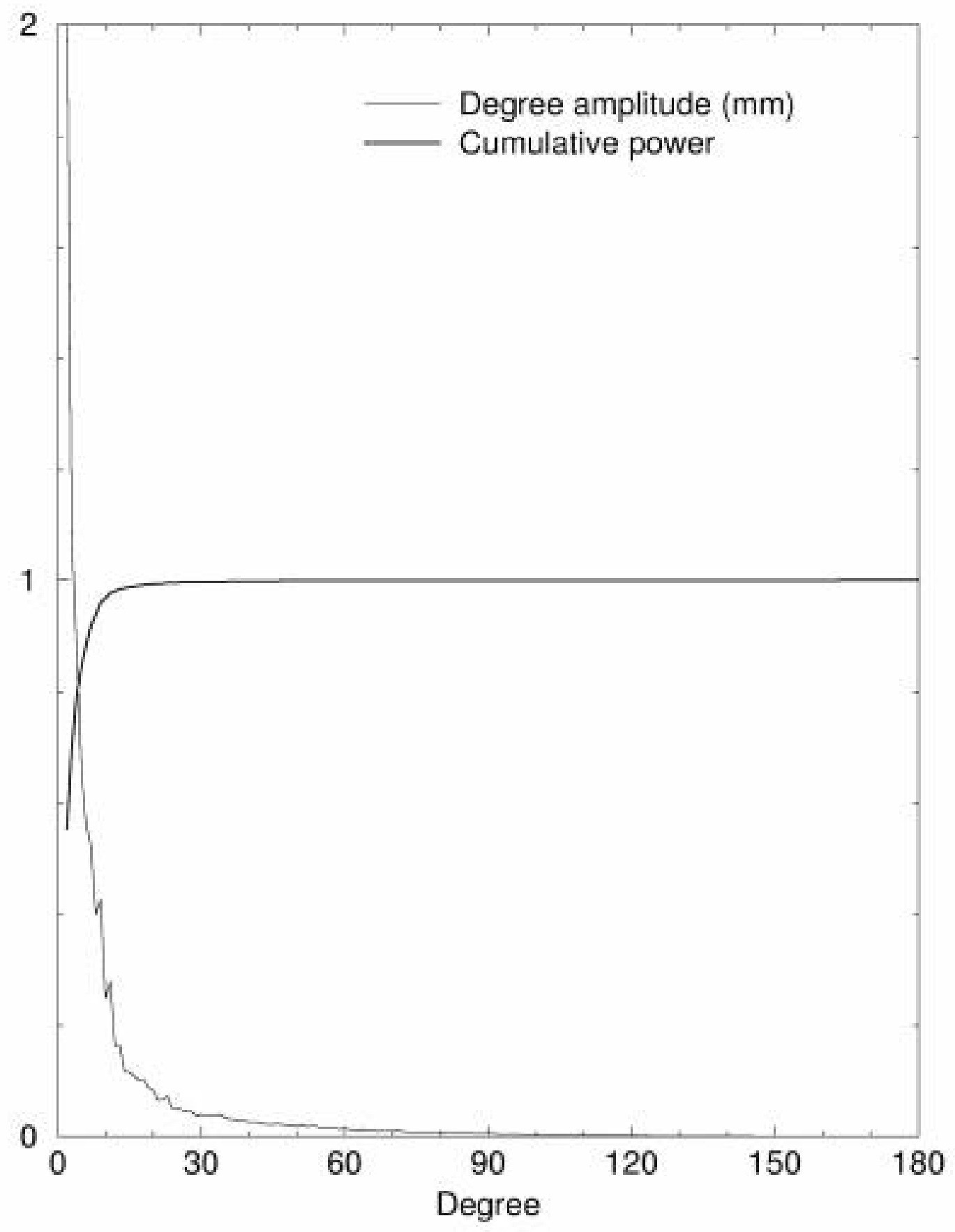

Fig. 10: Degree amplitude and cumulative percentage power of geoid variation computed from the corrected sea level anomaly in Fig. 9. 
At as low as degree 5, the signatures of geoid variation over the Pacific Ocean and the Indian Ocean are still apparent and are consistent with those of CSLA in Fig. 9. Specifically, in the western Pacific a negative CSLA results in a mass deficiency, which then causes a negative geoid variation there; in the eastern Pacific, the sign of CSLA is reversed, leading to a positive geoid variation there. During the 1997-1998 El Niño the largest geoid change occurred around the Galapagos Island and exceeded $1 \mathrm{~cm}$. Furthermore, by least-squares fitting an expression $\alpha_{n}-\beta$ to the averaged degree variances of the gravity variation, we find

$\bar{\tau}_{n}^{2}=\frac{1}{2 n+1} \sum_{m=0}^{n}\left(\bar{J}_{n m}^{2}+\bar{K}_{n m}^{2}\right) \approx 7 \times 10^{-19} n^{-4}$

Fig.12 compares the modeled and true degree variances of the gravity variation. Comparing the expressions in (34) and (40), we find

$$
\bar{\sigma}_{h} \approx 10^{4} \bar{\tau}_{n}
$$

Thus at any degree the static gravity signals (e.g., geoid undulation, gravity anomaly, deflection of the vertical etc.) are about 10000 times larger than the temporal gravity signals due to oceanic mass variation.

\subsubsection{Recovery}

Now we attempt to recover the geoid variation in Fig. 11 from the COSMIC data. For this simulation, we assume that EGM96 is the true, static gravity field determined from dedicated gravity missions such as GRACE, CHAMP and GOCE (Balmino et al., 1998). The timedependent gravity field is then the sum of EGM96 implied static gravity and the change of gravity. Let $\bar{C}_{n m}^{E}$ and $\bar{S}_{n m}^{E}$ be the geopotential coefficients of EGM96. The steps of simulation are:

(1) Compute $\bar{C}_{n m}^{T}=\bar{C}_{n m}^{E}+\bar{J}_{n m}$ and $\bar{S}_{n m}^{T}=\bar{S}_{n m}^{E}+\bar{K}_{n m}$

(2) Integrate 7-day orbits at a 1-minute interval using $\bar{C}_{n m}^{T}$ and $\bar{S}_{n m}^{T}$ up to degree 50 for each of the 8 COSMIC satellites. Random errors are added to the orbits based on a $3-\mathrm{cm}$ standard deviation. These orbits are treated as positional data of COSMIC.

(3) Repeat (2), but using $\bar{C}_{n m}^{E}$ and $\bar{S}_{n m}^{E}$

(4) Subtract the orbits in (3) from the orbits in (2) to get the radial, along-track and cross-track perturbations due to mass variation.

(5) Compute $\hat{\bar{J}}_{n m}$ and $\widehat{\bar{K}}_{n m}$, which are the estimates of $\bar{J}_{n m}$ and $\bar{K}_{n m}$, by least-squares using the linear perturbation theory with data from the "observed" perturbations in Step (4).

Fig. 13 shows the perturbations of a COSMIC satellite orbit due to the mass variation (note: no noises are added to these perturbations). If the higher order effects are removed, the RMS 


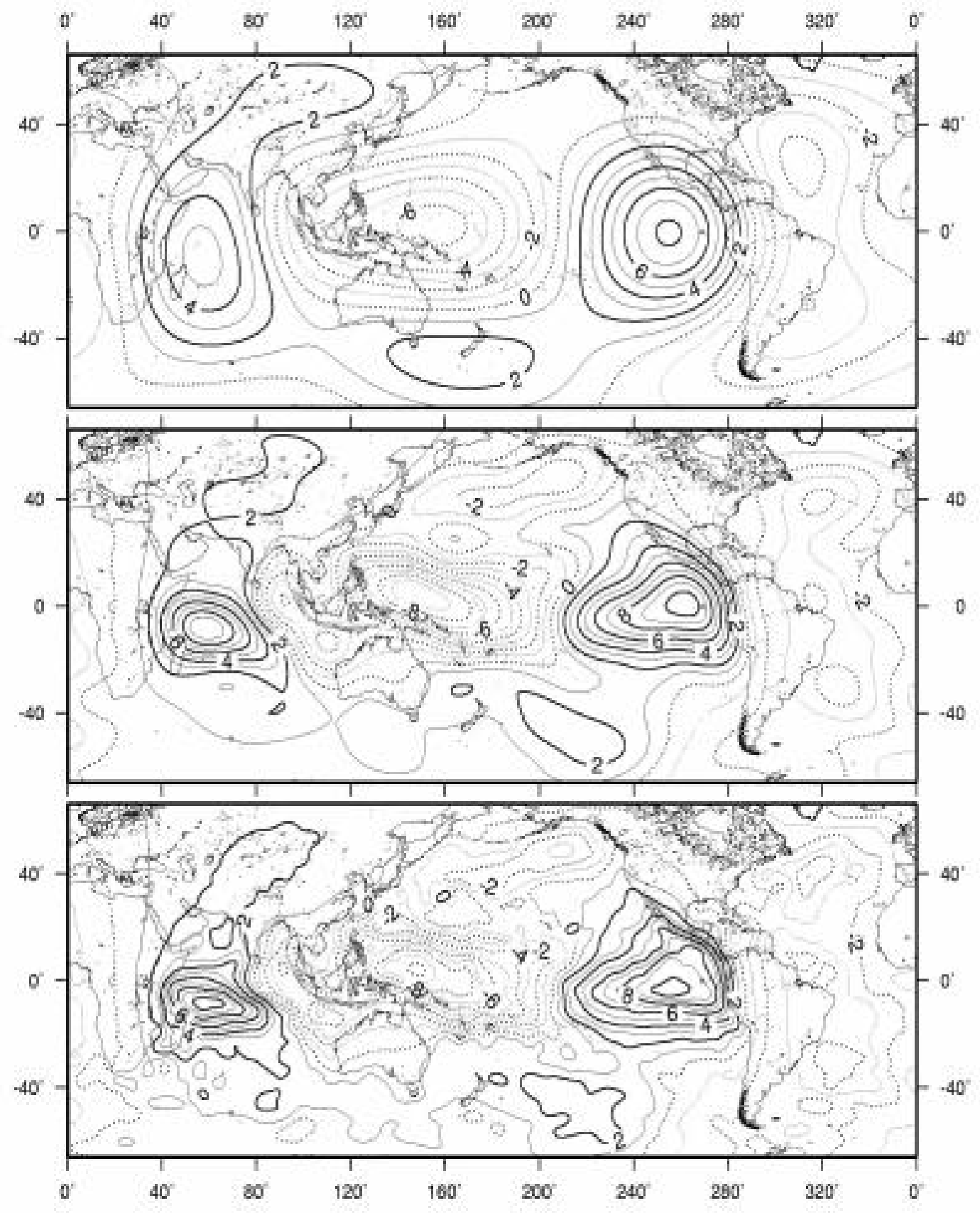

Fig. 11: Contour maps of geoid variation up to degrees 5 (top), 15 (center) and 50 (bottom) from the corrected sea level anomaly in Fig. 9. Unit is $\mathrm{mm}$. 


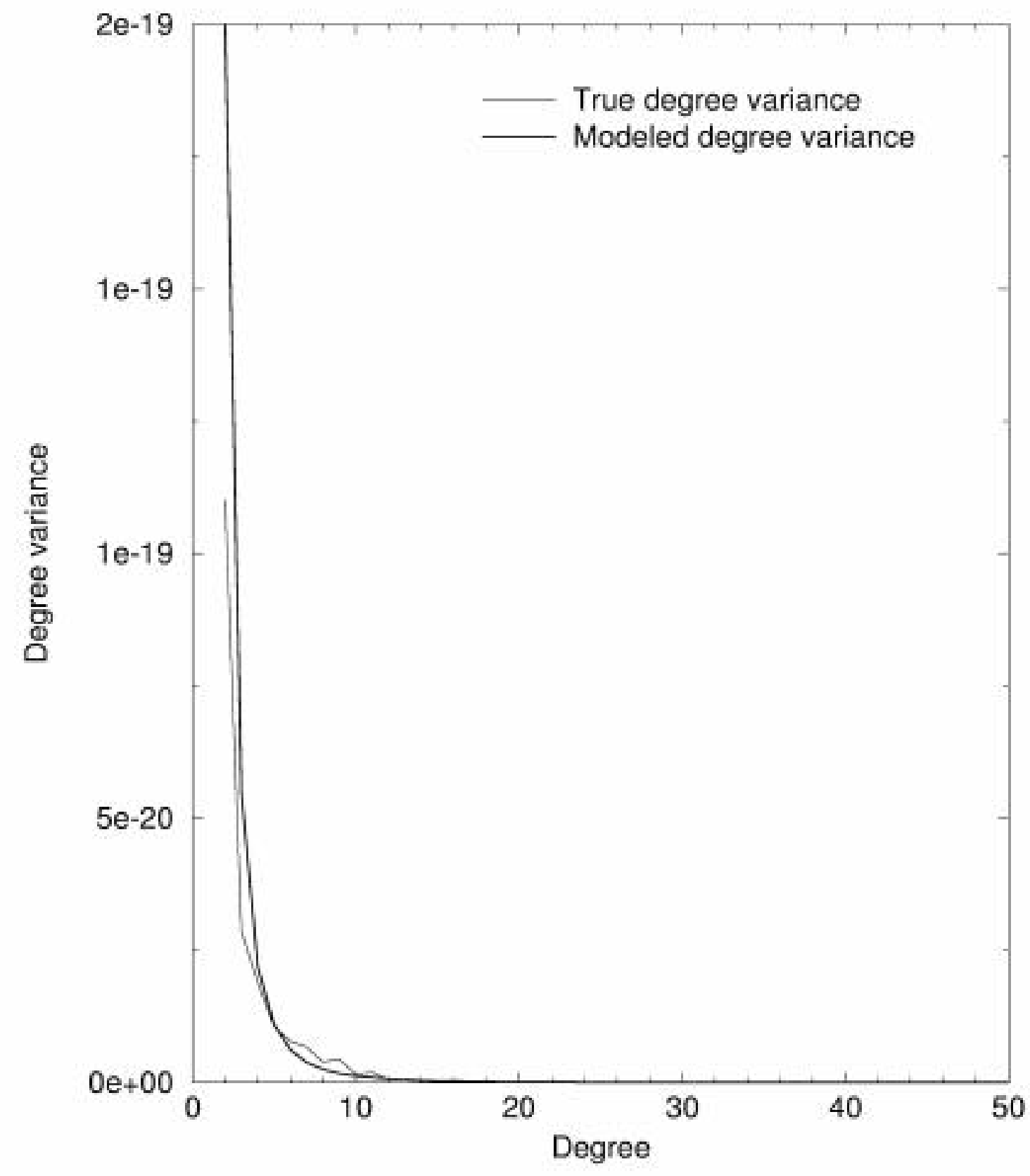

Fig. 12: True and modeled degree variances of gravity variation due to oceanic mass variation. 
values of the radial, along-track and cross-track perturbations are only $0.4,4.5$ and $1.2 \mathrm{~cm}$, respectively, which are very small and can be easily obscured by GPS noises. In the least-squares estimation, we have tried three different solutions: the first solution does not use any weighted constraints, the second solution uses constraints based on the static degree variances in (34), and the third solution uses constraints based on the temporal degree variances in (40). Unfortunately, in the first and second solutions the normal matrices were singular and hence the estimations were unsuccessful.

Fig. 14 shows the relative errors of the recovered coefficients from 7 days of COSMIC data from the third solution. Up to degree 18 the sectorial harmonics are best recovered and the low degree terms have relatively small errors. For most of the coefficients, the relative errors are below 1, indicating that the COSMIC satellites can sense harmonic coefficients up to as high as degree 50. Fig. 15 shows the recovered geoid variations up to degrees 5, 15 and 50. Due to the GPS noises and the polar gaps in the data coverage, the true geoid variations in Fig. 11 cannot be fully recovered. In addition, a surface signal such as geoid variation will be attenuated at satellite altitude, making a full recovery of the signal even more difficult. In fact, beyond degree 15 the resolution of the recovered geoid variation does not improve at all. We also tried a solution using one month of COSMIC data, but the result is not improved and is almost identical to that from one week of data. However, important signatures of the temporal gravity variation are retained in the recovered fields. For example, the geoid lows northeast of Australia and in the Atlantic Ocean, and the geoid highs near the Galapagos Island and east of Madagascar are clearly seen in Fig.15. Based on this experiment, we conclude that, given data noise of $3 \mathrm{~cm}$ at one-minute interval, COSMIC is able to see temporal gravity variation on time scale of at least one week and on spatial scale of about $2600 \mathrm{~km}$ (the equivalent scale of degree 15).

\subsubsection{The effect of data noise on recovering temporal gravity}

The result in the above experiment has shown that we cannot fully recover the temporal gravity variation with a $3-\mathrm{cm}$ noise in the COSMIC data. To see more about how the noise of COSMIC data will affect the result, we generated 7 days of orbit for each of the 8 COSMIC satellites using degree-50 fields in the orbit integrations as in the previous section. However, here we used three different noises in the "observed" perturbations: 3,1 and $0.1 \mathrm{~cm}$. The "observed" perturbations were then used to compute the temporal gravity variations up to degree 15 . The reasons for using the degree-15 solutions are: (1) to avoid aliasing into low-degree terms from the high-degree terms due to the polar gaps, (2) to avoid using any a priori weighted constraints of any kind, and (3) to avoid singularity in the normal matrix in case of no constraints. Fig. 16 shows the relative errors from using the three noises. Clearly the relative error decreases as the noise decreases. The result from the case with noise $=3 \mathrm{~cm}$ is almost identical to the result from the degree-50 solution (up to degree 15) described in the previous section. The best result is with noise $=0.1 \mathrm{~cm}$, and the recovered geoid variations up to degrees 5,10 and 15 are shown in Fig.17. The degree-10 geoid in Fig. 17 closely resembles the degree-15 geoid in Fig.11. In the case of $0.1-\mathrm{cm}$ noise, the degree-15 geoid improves the resolution over the degree-10 geoid, but the former contains some artifacts, e.g., the distorted geoid variations in the eastern Pacific and in the central Atlantic. We also find that the degree-5 geoid from the case with noise $=1 \mathrm{~cm}$ (not plotted here) agrees very well with the degree-5 geoid in Fig. 11. This experiment suggests 


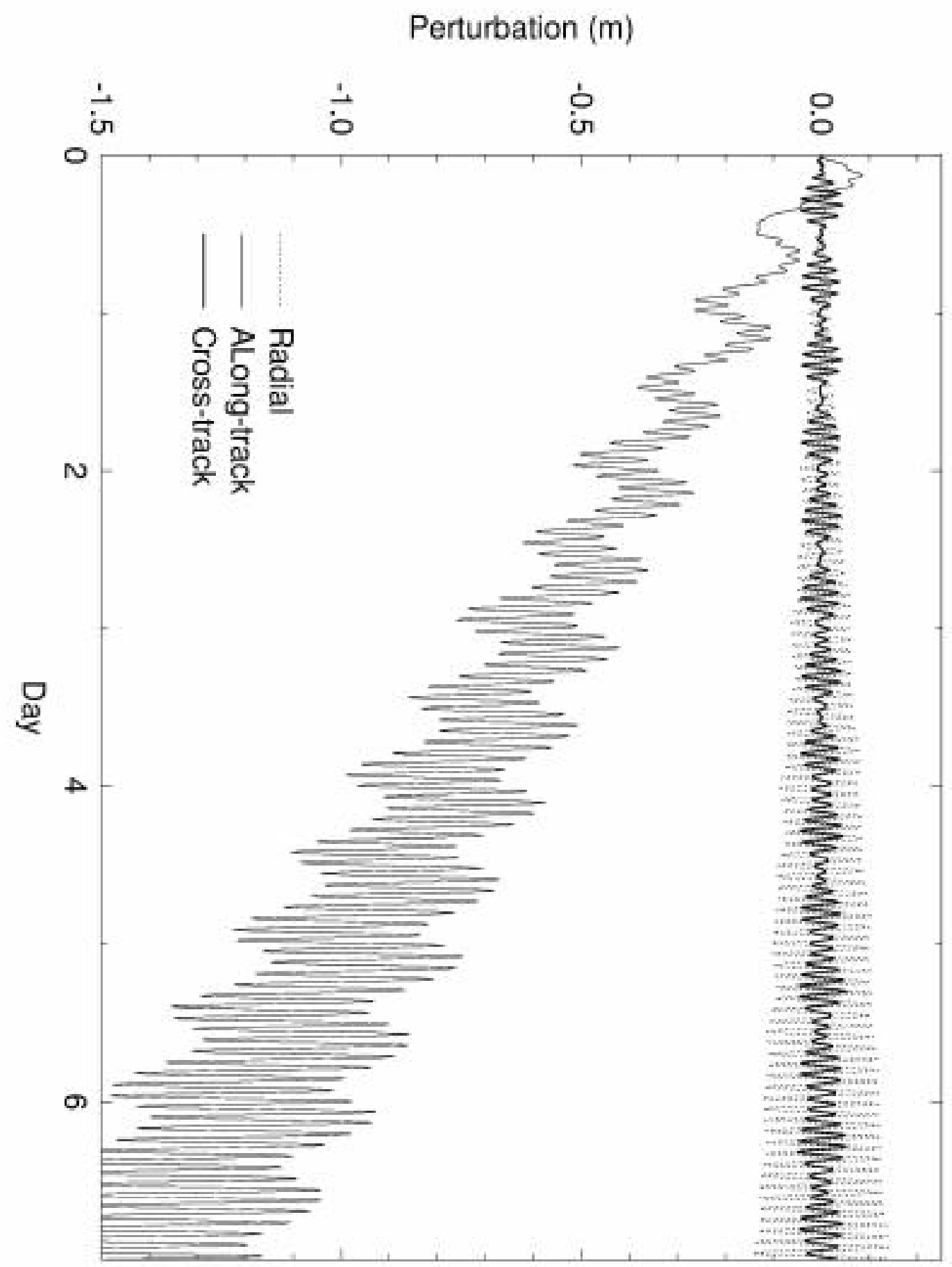

Fig. 13: Perturbations of COSMIC orbit due to the mass variation of corrected sea level anomaly up to degree 50. Day is the elapsed number of days since the first data point. 

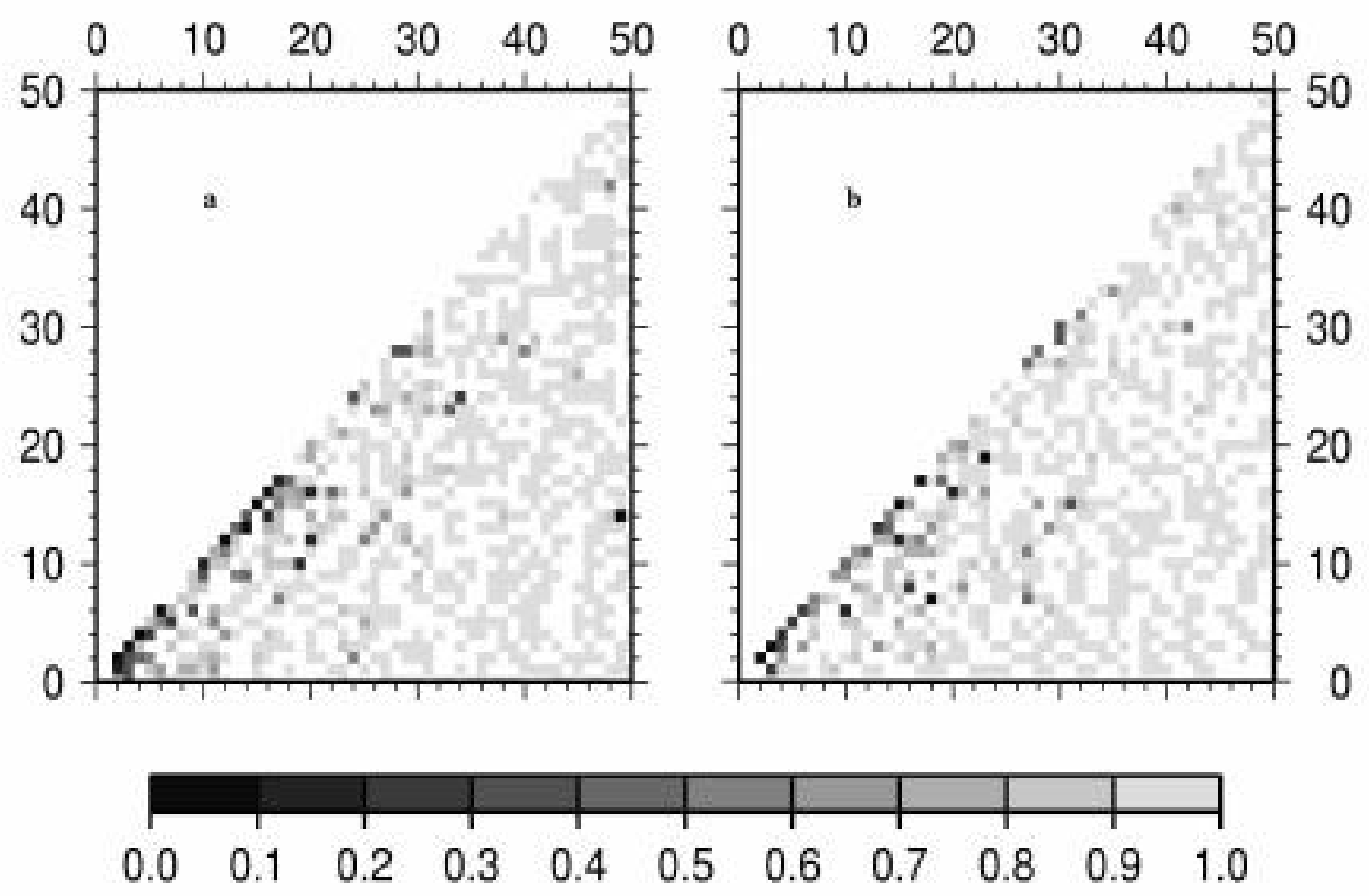

Fig. 14: Relative errors of the recovered harmonic coefficients of gravity variation for (a) $\hat{\bar{J}}_{n m}$ and (b) $\widehat{K}_{n m}$ using one week of COSMIC data and degree-50 solution. 


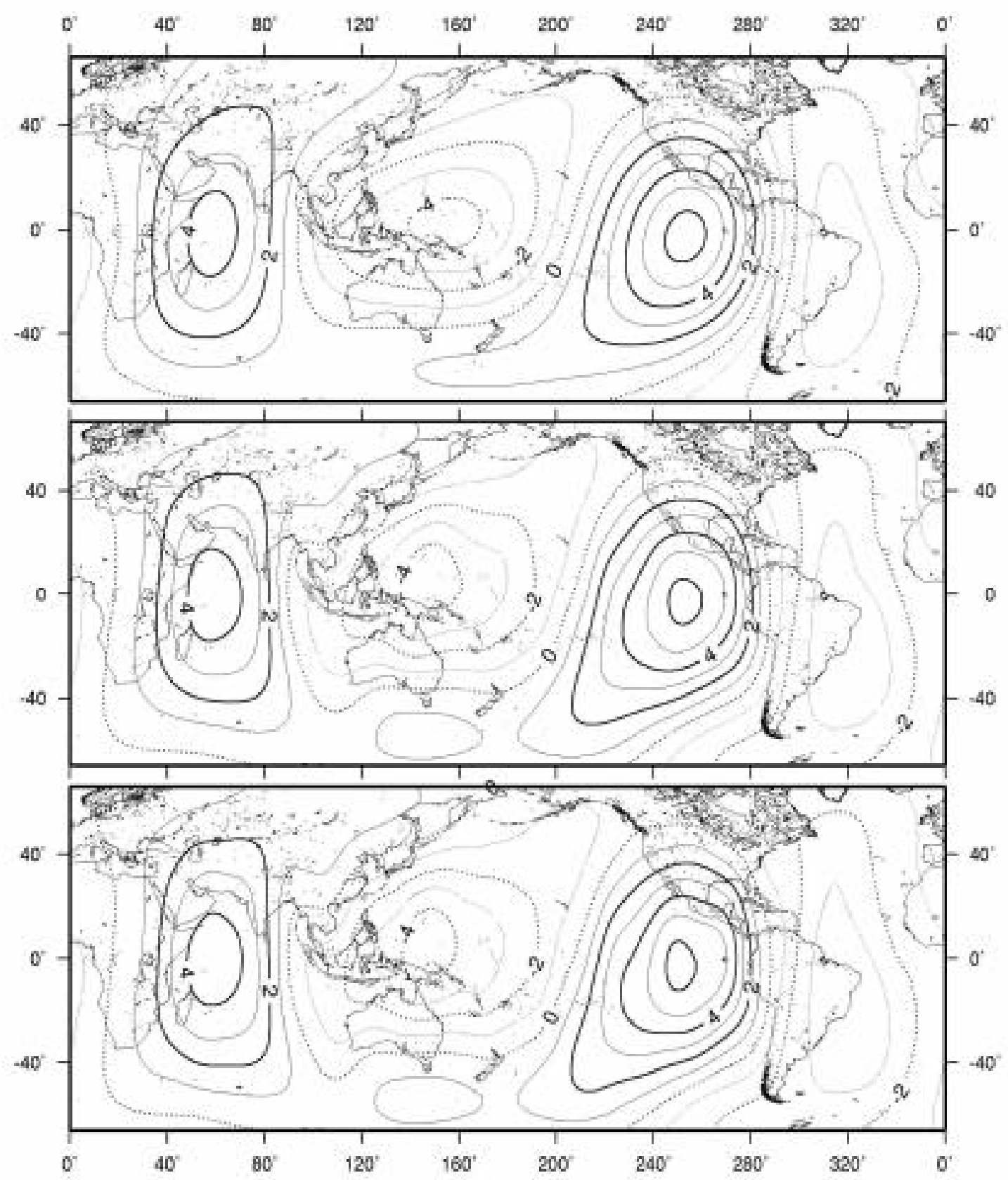

Fig. 15: Contour maps of recovered geoid variation up to degrees 5 (top), 15 (center) and 50 (bottom) using one week of COSMIC data and degree-50 solution. Unit is mm. 
that the noise of COSMIC data has a substantial impact on the achievable resolution in the recovered gravity variation. Furthermore, it appears that the polar gaps in the COSMIC orbits will not do too much damage to the low-degree solutions performed here, but it is expected that the accuracy of recovery will be improved if the COSMIC satellites are in polar orbits. 


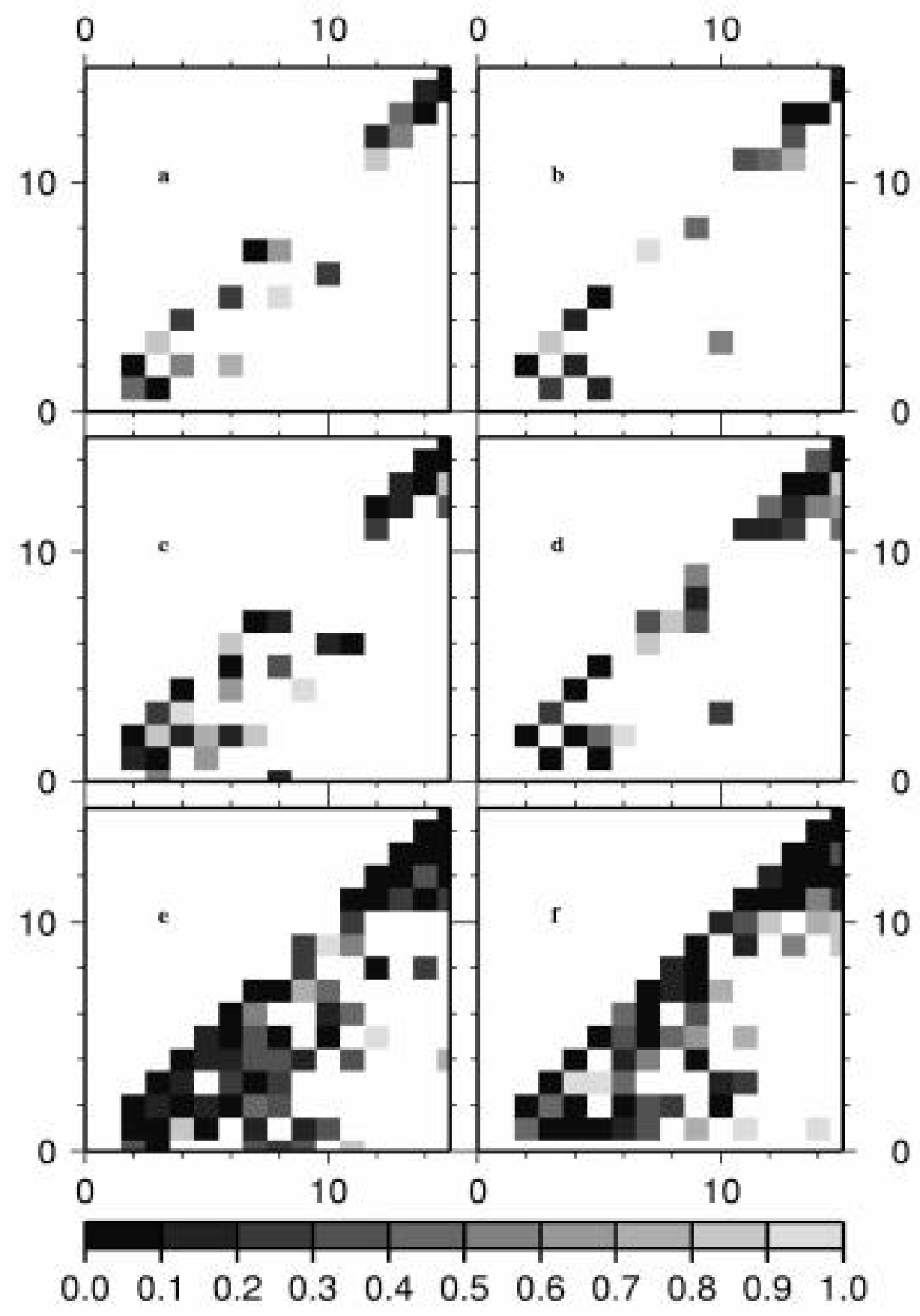

Fig. 16: Relative errors of the recovered harmonic coefficients of gravity variation using one week of COSMIC data and degree-15 solutions for (a) $\hat{\bar{J}}_{n m}$ (3-cm noise) (b) $\widehat{\bar{K}}_{n m}$ (3-cm noise) (c) $\hat{\bar{J}}_{n m}$ (1-cm noise) (d) $\widehat{\bar{K}}_{n m}$ (1-cm noise) (e) $\hat{\bar{J}}_{n m}\left(0.1-\mathrm{cm}\right.$ noise) (f) $\widehat{\bar{K}}_{n m}$ (0.1-cm noise). 


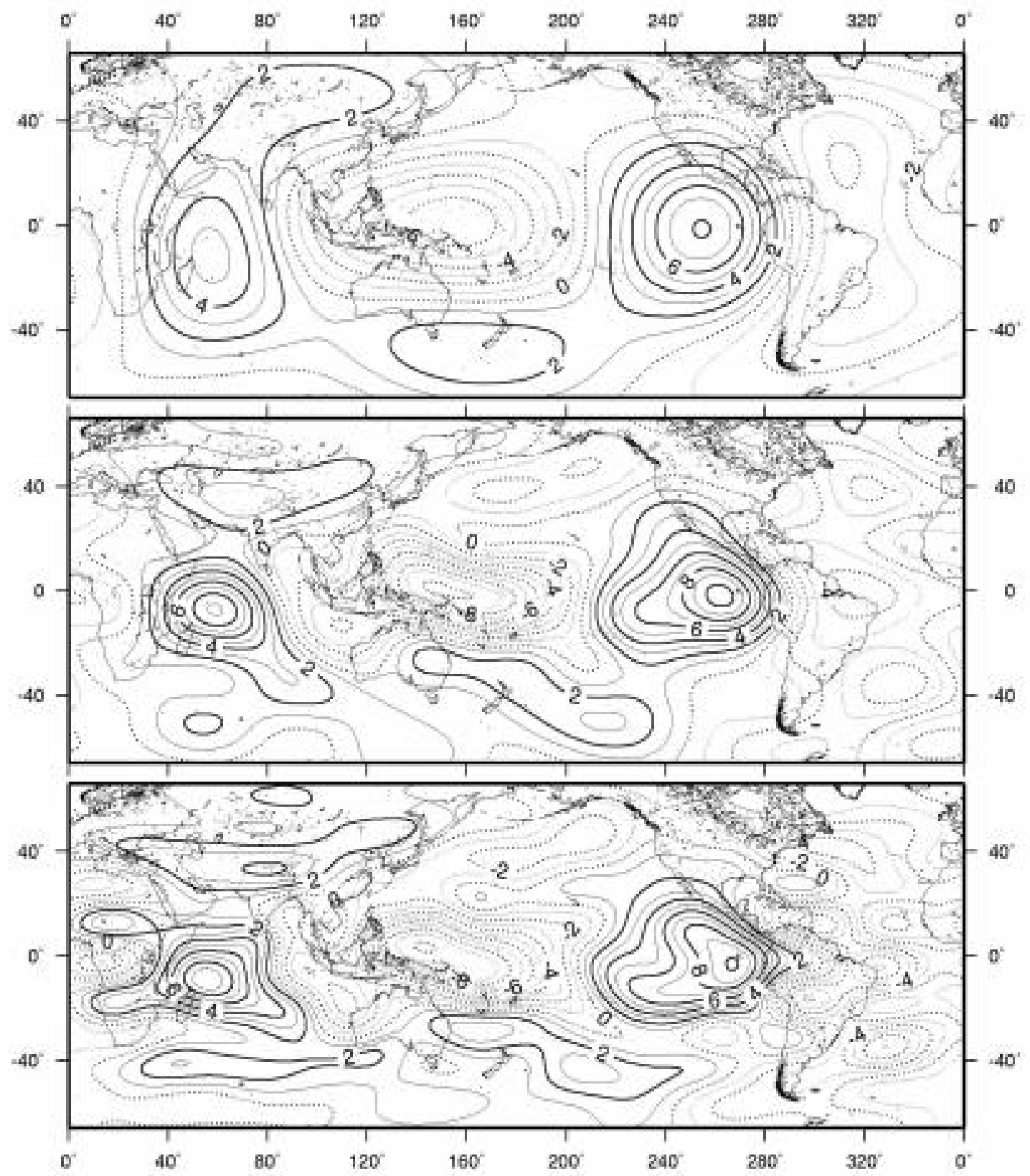

Fig. 17: Contour maps of recovered geoid variation up to degrees 5 (top), 10 (center) and 15 (bottom) using one week of COSMIC data and degree-15 solution with noise $=0.1 \mathrm{~cm}$. Unit is $\mathrm{mm}$. 


\section{Conclusion and recommendation}

In this study we proposed improved formulae of orbital perturbations, which were then verified by numerical analysis. The perturbation formula were used to recover gravity fields using the simulated, GPS-derived positional data from the COSMIC mission in the operational phase. Our results show that the COSMIC data can improve the long wavelength part of the EGM96 model. Most important is that the COSMIC data can be used to recover temporal gravity variations, especially the gravity signatures due to the mass movement in an El Niño. Our experiments also show that to see clearly the structure of temporal gravity variation, the noise of COSMIC positional data should be reduced to $1 \mathrm{~cm}$ or less at the one-minute sampling interval. Thus, it is necessary to develop a good data processing method for noise reduction. The technique developed here can be applied to gravity recoveries from the data of the CHAMP and GRACE missions, which have onboard accelerometers to measure surface forces. With the measured surface forces, there will be no need to use a priori force models in our approach and the result should be improved. This study has focused on the use of positional data of COSMIC. A different use of the COSMIC GPS data for gravity recovery is possible and this is a subject for future study. 


\section{Appendix A: Order-zero perturbations}

For a satellite orbit with a small eccentricity, we may assume $r=a, f=E=M$ in (4), (5) and (6) to obtain the approximations:

$\Delta x_{1}^{0}=(1-e \cos M) \Delta a-(a \cos M) \Delta e+(a e \sin M) \Delta M$

$\Delta x_{2}^{0}=a[\Delta \omega+\Delta M+(\cos I) \Delta \Omega$

$\Delta x_{3}^{0}=a[(\sin (\omega+M)) \Delta I-(\sin I \cos (\omega+M)) \Delta \Omega$

Furthermore, in (14) we set $Q=1$. This truncated series of $R$ will then require only the $G_{n p 0}, G_{n p \pm 1}$ terms, which can be approximated as (Balmino, 1994, p. 270)

$$
\begin{aligned}
G_{n p 0} & =1, G_{n p 1}=\frac{(3 n-4 p+1) e}{2}, G_{n p-1}=\frac{(-n+4 p+1) e}{2} \\
G_{n p 0}^{\prime} & =0, \quad G_{n p 1}^{\prime}=\frac{3 n-4 p+1}{2}, G_{n p-1}^{\prime}=\frac{-n+4 p+1}{2}
\end{aligned}
$$

Substituting (A-4) and (A-5) into (20), (19), and finally into (A-1), (A-2) and (A-3), with some trigonometric identities we obtain the order-zero perturbations

$$
\begin{aligned}
& \Delta x_{1}^{0}=\sum_{n=2}^{K} \sum_{m=0}^{n} \sum_{p=0}^{n} C_{n m p}^{1} S_{n m p 0} \\
& \Delta x_{2}^{0}=\sum_{n=2}^{K} \sum_{m=0}^{n} \sum_{p=0}^{n} C_{n m p}^{2} S_{n m p 0}^{*} \\
& \Delta x_{3}^{0}=\sum_{n=2}^{K} \sum_{m=0}^{n} \sum_{p=0}^{n}\left(C_{n m p}^{3-} S_{(n+1) m p 0}^{*}-C_{n m p}^{3+} S_{(n-1) m p 0}^{*}\right.
\end{aligned}
$$

where

$$
\begin{aligned}
C_{n m p}^{1} & =\bar{n} a\left(\frac{a_{e}}{a}\right)^{n} \bar{F}_{n m p}\left[\frac{2(n-2 p)}{\dot{\psi}_{n m p 0}}+\frac{-3 n+4 p-1}{2 \dot{\psi}_{n m p 1}}+\frac{-n+4 p+1}{2 \dot{\psi}_{n m p-1}}\right] \\
C_{n m p}^{2} & =\bar{n} a\left(\frac{a_{e}}{a}\right)^{n} \bar{F}_{n m p}\left[\frac{2(n+1)-3(n-2 p) \bar{n} / \dot{\psi}_{n m p 0}}{\dot{\psi}_{n m p 0}}+\frac{-3 n+4 p-1}{2 \dot{\psi}_{n m p 1}}+\frac{n-4 p-1}{\dot{\psi}_{n m p 1}}\right]
\end{aligned}
$$




$$
\begin{gathered}
C_{n m p}^{3 \pm}=\frac{a}{2}\left(\frac{a_{e}}{a}\right)^{n} \frac{\bar{n}}{\psi_{h m p 0}}\left\{[(n-2 p) \cos I-m] \frac{\left.\bar{F}_{n m p} \pm F_{n m p}^{\prime}\right)}{\sin I}\right. \\
S_{(n \pm 1) m p 0}^{*}=\left[\begin{array}{c}
\bar{C}_{n m}^{+} \\
-\bar{S}_{n m}^{-}
\end{array}\right] \sin \psi_{(n \pm 1) m p 0}-\left[\begin{array}{c}
\bar{S}_{n m}^{+} \\
-\bar{C}_{n m}^{-}
\end{array}\right] \cos \psi_{(n \pm 1) m p 0}
\end{gathered}
$$

This result can also be found in Rosborough and Tapley (1987). 
Appendix B: Computation of steric anomaly due to thermal expansion

The steric anomaly is sea level variation due to the expansion or contraction of sea water as a result of temperature variations over the entire column of the oceans. However, the upper layers of the oceans contribute most to the steric anomaly. In practice we take into account the contributions from the upper 14 oceanic layers when computing the steric anomaly:

$$
\Delta H_{S}=\sum_{i=1}^{14} \alpha_{i} \Delta T_{i} H_{i}
$$

where $\alpha_{i}$ is the coefficient of thermal expansion from Gill [1982, Table A3.1], and $\Delta T_{i}$ is the temperature anomaly relative to the mean of layer $i$ and $H_{i}$ is the thickness of layer $i$; ; see also Chen et al. (2000, Table 1) for a list of depths and thicknesses of the 14 layers. We obtained via the Internet the monthly, $1^{\circ} \times 1^{\circ}$ gridded temperature data at different depths from the Integrated Global Ocean Services System (IGOSS) at the Columbia University (see the WWW site: http://lola.ldgo.columbia.edu/SOURCES/.LEVITUS94). Then, at each of the 14 layers, the mean temperature was determined and subtracted from the raw temperature to get the temperature anomaly $\Delta T_{i}$. It turns out that the quality of temperature data varies over space and time. So we use a medium filter with a $300-\mathrm{km}$ wavelength to filter the computed steric anomaly. Ideally we should compute the steric anomaly at the mean time of T/P Cycle 196, but the current maximum resolution of reliable global temperature data at different depths is probably only one month. 
Appendix C: Harmonic coefficients and geoid variation from corrected sea level anomaly

Steric anomaly-corrected sea level anomaly (CSLA) from satellite altimetry includes the deviation of the instantaneous sea surface (after removing ocean tide and other geophysical effects) from a mean sea surface, and the vertical loading deformation due to the mass of such a deviation. CSLA in this case may be expanded into a series of spherical harmonics as

$\left.\Delta h(\theta, \lambda)=\sum_{n=0}^{K}\left(1+k_{n}\right) \sum_{m=0}^{n} \bar{a}_{n m} \bar{R}_{n m}(\theta, \lambda)+\bar{b}_{n m} \bar{S}_{n m}(\theta, \lambda)\right]$

where $k_{n}$ is elastic Love number of degree $\mathrm{n}$ (for their numerical values, see, e.g., Wahr et al. (1998)), $\quad \bar{R}_{n m}=\bar{P}_{n m} \cos m \lambda, \bar{S}_{n m}=\bar{P}_{n m} \sin m \lambda$ are fully normalized spherical harmonics (Heiskanen and Moritz, 1985; see also (1)). The coefficients $\bar{a}_{n m}$ and $\bar{b}_{n m}$ are harmonic coefficients of CSLA without loading effect. and can be obtained by the integrations

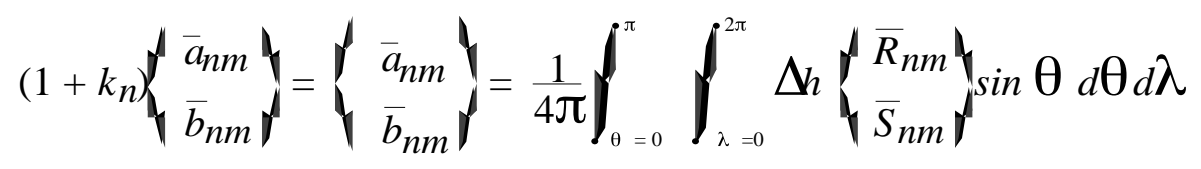

At any point exterior to the earth, the potential due to the mass of $\Delta h$ is

$$
\Delta V(r, \theta, \lambda)=R_{e} G \rho_{w} \int_{\theta^{\prime}=0}^{\pi} \int_{\lambda^{\prime}=0}^{2 \pi} \frac{\Delta h}{s} \sin \theta^{\prime} d \theta^{\prime} d \lambda^{\prime}
$$

where $\rho_{w} \approx 1.03 \mathrm{~g} \mathrm{~cm}^{-3}$ is the density of sea water, $R_{e} \approx 6371 \mathrm{~km}$ is the earth's mean radius, $G$ is the gravitational constant, and $s$ is the distance between the point at $(r, \theta, \lambda)$ and a mass element. The inverse of $s$ can be expanded into products of spherical harmonics as (Heiskanen and Moritz, 1985)

$$
\frac{1}{s}=\frac{1}{r} \sum_{n=0}^{\infty} \frac{1}{2 n+1}\left(\frac{R}{r}\right)^{n} \sum_{m=0}^{n}\left[R_{n m}\left(\theta^{\prime}, \lambda^{\prime}\right) \bar{R}_{n m}(\theta, \lambda)+\bar{S}_{n m}\left(\theta^{\prime}, \lambda^{\prime}\right) \bar{S}_{n m}(\theta, \lambda)\right]
$$

Substituting (C-4) and (C-1) into (C-3) and using the orthogonal relationship of spherical harmonics, we get

$$
\Delta V(r, \theta, \lambda)=\frac{G M}{r} \sum_{n=0}^{\infty}\left(\frac{R_{e}}{r}\right)^{n} \sum_{m=0}^{n}\left[\overline{\mathbf{I}}_{n m} \bar{R}_{n m}(\theta, \lambda)+\bar{K}_{n m} \bar{S}_{n m}(\theta, \lambda)\right]
$$

where $M$ is the earth's mass and

$$
\left.\left\{\begin{array}{l}
\bar{J}_{n m} \\
\bar{K}_{n m}
\end{array}\right\}=\frac{4 \pi \rho_{w} R^{2}\left(1+k_{n}\right)}{M(2 n+1)}\left\{\begin{array}{c}
\bar{a}_{n m} \\
\bar{b}_{n m}
\end{array}\right\}=\frac{4 \pi \rho_{w} R_{e}^{2}}{M(2 n+1)} \backslash \bar{a}_{n m}^{\prime} \bar{b}_{n m}^{\prime}\right\}
$$


Thus the elastic Love numbers are canceled out. The harmonic coefficients of $\Delta V$ differ from those of CSLA by only a scale factor. Let $C_{n m}=\left(a_{n m}^{\prime}+i \bar{b}_{n m}^{\prime}\right)$, where $i=\sqrt{-1}$. Given $\Delta h$ on a regular $\Delta \theta \times \Delta \lambda$ grid, (C-5) can be approximated as

$$
\begin{aligned}
C_{n m} & =\frac{1}{4 \pi q_{n}} \sum_{k=0}^{M-1} \sum_{l=0}^{N-1} \overline{\Delta h}\left(\theta_{k}, \lambda_{l}\right)\left[\int_{t k}^{t k+1} \bar{P}_{n m}(t) d t\right]\left[\int_{\lambda t}^{\lambda t+1} e^{-i m \lambda} d \lambda\right] \\
& =\frac{g m}{4 \pi q_{n}} \sum_{k=0}^{M-1} I \bar{P}_{n m}^{k} \sum_{l=0}^{N-1} \overline{\Delta h}\left(\theta_{k}, \lambda_{l}\right) e^{i 2 \pi m l / N}
\end{aligned}
$$

where $t_{k}=\cos (k \Delta \theta), \lambda_{l}=l \Delta \lambda, I \bar{P}_{n w}^{k}$ is the integration of the associated Legendre function (Paul, 1978), $q_{n}$ is a smoothing factor defined by Rapp (1989, p. 266), and

$$
g_{m}=\left\{\begin{array}{c}
\Delta \lambda \text { if } m=0 \\
{[\sin (m \Delta \lambda-\text { i }(\cos (m \Delta \lambda-1)] / m, \text { if } m \neq 0}
\end{array}\right.
$$

$$
\overline{\Delta h}\left(\theta_{k}, \lambda_{l}\right)=\frac{1}{4}\left[\Delta h\left(\theta_{k}, \lambda_{l}\right)+\Delta h\left(\theta_{k+1}, \lambda_{l}\right)+\Delta h\left(\theta_{k}, \lambda_{++1}\right)+\Delta h\left(\theta_{k+1}, \lambda_{+1}\right)\right]
$$

Thus $\Delta \bar{h}$ is the simple mean of four neighboring point values. The expression $\sum_{l=0}^{N-1} \overline{\Delta h}\left(\theta_{k}, \lambda_{l}\right) e^{i 2 \pi m l / N}$ in (C-7) can be computed efficiently by FFT. With $C_{n m}$ computed, $\bar{a}_{n m}^{\prime}$ and $\bar{b}_{n m}^{\prime}$ are simply taken from its real and imaginary parts, respectively.

The geoid variation due to $D h$ is simply computed by Bruns' formula $\Delta N=\Delta V / \gamma$, where $\gamma$ is normal gravity. On sea level, we can set $r=R_{e}$ and $\gamma=G M / R_{e}^{2}$ to get the geoid variation by

$$
\left.\Delta N(\theta, \lambda)=R_{e} \sum_{n=0}^{\infty} \sum_{m=0}^{n} \overline{\mathbf{J}}_{n m} \bar{R}_{n m}(\theta, \lambda)+\bar{K}_{n m} \bar{S}_{n m}(\theta, \lambda)\right]
$$

The error in such a spherical approximation is very small because the geoid variation is already very small. 


\section{APPENDIX D: Important FORTRAN programs}

This appendix lists important FORTRAN programs developed in this study. The programs are written in a fashion that all I/O parameters can be specified online. To carry out a specific job described in this report, all needed programs (executables) can be collected in one batch file. The batch file is then submitted under the UNIX environment to finish all needed computations in one single run. All FORTRAN programs listed below have the suffix .f.

\section{degvar.f}

USAGE:

degvar coef_file -Llmax -Nname -Ttype [-E -Sscale]

OPTIONS:

coef_file file of geopotential coefficients

-L max harmonic degree

$-\mathrm{N}$ name of output variance and squared root variance

-T type of degree variance or error degree variance

$0=$ geoid, $1=$ gravity anomaly, $2=$ geopotential

-E compute error degree variance [default; degree variance]

-S scale factor [default: 1]

DESCRIPTION:

degvar.f computes degree variances or degree variances of a geopotential model.

\section{detrend.f}

USAGE:

OPTIONS:

detrend -Apert.rtn -Bpert_rm.rtn -Ctrend.rtn

-A file of radial, along-track, cross-tack perturbations

-B same as -B, but the perturbations are detrended using empirical models

$-\mathrm{C}$ same as $-\mathrm{B}$, but the contain the trends in the three perturbations

\section{DESCRIPTION:}

detrend.f removes the higher order effects in the radial, along-track, cross-tack perturbations

\section{diffcoef.f}

USAGE:

diffcoef -Acoef1 -Bcoef2 -Cname -Llmax [-R -T -Z]

OPTIONS:

-A first set of coefficients

$-\mathrm{B}$ second set of coefficients

-C difference, (A-B)

- $\mathrm{L}$ max degree of comparison

$-\mathrm{R}$ write relative errors abs ((A-B)/A) [default: No] in .grd1 format

-T minimum acceptable relative error in .grd1

$-Z$ write relative errors of zonal harmonics

DESCRIPTION: 
diffcoef.f computes the statistics of the difference between two sets of geopotential coefficients

\section{integr.f}

USAGE:

integr -Cgfile -Eefile -Tstop_time -Oiner.xyz -Lnmax -Ddelt

[-Ggeod.xyzt -K]

OPTIONS:

-C file of geopotential coefficients, including GM, and a

-L max degree of geopotential under consideration

-E file of start time and the initial state vector

-T file of stop time in year, month, day, hour, min, sec

-D increment of orbit in output file in seconds (for -O)

-O output file of time, $\mathrm{x}, \mathrm{y}, \mathrm{z}, \mathrm{vx}, \mathrm{vy}$, vz. time is in MJD

-G output file of longitude, latitude, ellipsoidal height and time [default: no such output]

-K the initial state vector contains Keplerian elements. the order

is: a, e, i, argument of perigee, right ascension of ascending node, mean anomaly [default: rectangular elements]

DESCRIPTION:

integr.f integrates satellite orbit perturbed by the earth's nonsphericity using the Cowell II formulation of EOM with the predict- psuedo-correct algorithm. DVDQ is used as the numerical integrator. The usage of DVDQ can be found in Krogh (1969). Assume (1) UT1-UTC $=0$ (2) no precession, nutation and polar motion.

\section{intorb.f}

USAGE:

intorb -Adata_orbit -Btimes_int [-Ddegree -Pno_varables -E]> out_orbit OPTIONS:

-A orbit file with equally-spaced coordinates and/or velocities

format: time, $[\mathrm{x}, \mathrm{y}, \mathrm{z}, \mathrm{Vx}, \mathrm{Vy}, \mathrm{Vz}, \ldots]$

-B file of times where interpolations are wanted

format: time (same unit as -A time)

-D polynomial degree [default: 14]

-P number of dependent variables to be interpolated[default: 6]

-E exclude interpolated values at the two end zones, will lose data [default: do not exclude end zones]

out_orbit output file (same format as -A file)

\section{DESCRIPTION:}

intorb.f interpolates satellite positions and velocities using polynomials. The data are assumed to be equally spaced in time.

\section{norm.f}

USAGE:

norm -Oobs -Nnu.mat -Llmax -Tstart/stop 
OPTIONS:

-O file of t,a,e,i,w,om,ma,r,t,n (the last three are observations), $\mathrm{t}$ is in $\mathrm{mjd}$

$-\mathrm{N}$ file of normal matrix and $\mathrm{u}$-vector. $\mathrm{NX}=\mathrm{U}$, where $\mathrm{X}$ is the vector of unknowns

-L max harmonic degree of the solution

-T start/stop times of the used arc in mjd

DESCRIPTION:

norm.f forms normal equations of spherical harmonic coefficients of geopotential and other parameters using GPS-determined positions of an earthorbiting satellite and the order-zero formulae of linear Parameters under considerations include: (1) spherical up to degree LMAX, excluding C00,C10,C11,S11, (2) perturbations. for the higher order effects.

\section{norm_t2.f}

USAGE:

norm_t2 -Oobs.rtn -Nnu.mat -Llmax -Tstart/stop [-Ptol -Qqmax -Sn -W]

OPTIONS:

-O file of observarions

$-\mathrm{N}$ file of normal matrix and $\mathrm{u}$-vector. $\mathrm{NX}=\mathrm{U}$, where $\mathrm{X}$ is the vector

of unkowns

-L max harmonic degree of the solution

-T start/stop times of the used arc in $\mathrm{mjd}$.

-P tolerance number of resonance effect [default: 0.01$]^{\prime}$

-Q max of q index in the eccentricity function [default:1]'

$-\mathrm{S}$ use every nth observation to accumulate normal matrix [default: 1]

-W data noise is unknown, all data have identical weight

\section{DESCRIPTION:}

harmonic coefficients

empirical coefficients

norm_t2.f has the same function as norm.f, i.e., it forms normal equations of spherical harmonic coefficients of the geopotential and empirical parameters using GPSdetermined positions of an earth-orbiting satellite. But the perturbation formulae are based on those developed in this paper.

\section{perturb.f}

USAGE:

perturb -Ccoeff -Oorbit -Eperturb -Llmax -Tstart/stop [-Ptol]

\section{OPTIONS:}

$-\mathrm{C}$ file of difference of geop. coeff.

$-\mathrm{O}$ file of elements of mean orbit and Kepler elements

-E file of radial, transverse and normal orbit perturbations

-L maximum degree of geopotential coefficients under consideration

-T start/stop times of the orbit arc in mjd.

$-\mathrm{P}$ tolerance of frequency ratio with one $\mathrm{cpr}$. tol $>=0.01$ in order to have a meaningful result [default: 0.01 ] 


\section{DESCRIPTION:}

perturb.f computes the radial,along-track and cross-tracks perturbations

due to the geopotential using order-zero formula.

\section{perturb_t2.f}

USAGE:

perturb -Ccoeff -Oorbit -Eperturb -Llmax -Tstart/stop

[-Ptol -Qqmax]

$-\mathrm{C}$ file of difference of geopotential coefficients

$-\mathrm{O}$ file of elements of mean orbit and Kepler elements

-E file of radial, transverse and normal orbit perturbations

-L maximum degree of geopotential coefficients unde consideration

-T start/stop times of the used arc in mjd

$-\mathrm{P}$ tolerance of frequency ratio with one cpr. tol $>=0.01$ in order to have a meaningful result [default: 0.01 ]

$-\mathrm{Q}$ max of q index in the eccentricity function [default:1]

\section{DESCRIPTION:}

Same as perturb.f, but perturb.f uses the perturbation formulae developed in this paper.

rec2rtn.f

USAGE:

rec2rtn -Atrue_orb -Bperturb_orb -Ooutput_file [-R -D

-Serr_r/err_t/err_n -H -E]

-A orbit A. format: time, $\mathrm{x}, \mathrm{y}, \mathrm{z}, \mathrm{Vx}, \mathrm{Vy}, \mathrm{Vz}$ in ASCII

-B orbit B. format same as true orbit

-O output file.

$-\mathrm{R}$ remove higer order effects

-D the unit of time is day [default: second]

-S std. dev. in meter added to $\mathrm{r}, \mathrm{t}, \mathrm{n}$.

-H ouput without first two records [default: with first two records containing mean orbit and std. dev. from $-\mathrm{S}]$

-E ouput time in seconds

\section{DESCIPTION:}

rec2rtn.f rotates the rectangular compoents to radial, along-track and crosstrack components using $(\mathrm{rt} \mathrm{n})=\mathrm{R}(\mathrm{dx} \mathrm{dy} \mathrm{dz})$ where $\mathrm{dx}, \mathrm{dy}, \mathrm{dz}$ are differences of orbit (B-A) in rectangular coordinates and $\mathrm{R}$ is a rotation matrix. The output_file will contain:

first record: averaged a,e,i second record: noises of rtn obs third to last records: time, $\mathrm{u}$, om and r,t,n.

This is for input to norm.f and perturb.f. time must be in mjd in this case.

\section{sha.f}

USAGE: 
sha grd_file -Lnmax -Ccoef_file [-Dr-Aa -Ttype -B]

grd_file input data file in .grd1 format

-L maximum degree of spherical harmonic expansion

$-\mathrm{C}$ ascii file of harmonic coefficients

-T type of data-coefficient relationship [default: 1]

-D radius on which the data are given [default: $6378136.3 \mathrm{~m}$ ]

-A radius on which the coefficients are defined [default: $6378136.3 \mathrm{~m}$ ]

-B request binary output of coefficients [default: ascii]

\section{DESCRIPTION:}

sha.f computes spherical harmonic coefficients from gridded ata on a sphere of radius

r. The data are in.grd 1 format and are over 0/360/-90/90. The available TYPE is:

Type data coefficient Remark

$\begin{array}{llll}1 & \text { SLA above ms } & \text { potential coeff } & r=a=6378136.3 \mathrm{~m} \\ 2 & \text { arbitrary function } & \text { coeff of function } & \mathrm{r}=\mathrm{a}=6378136.3 \mathrm{~m} \\ 3 & \text { grav anom on mss } & \begin{array}{c}\text { potential coeff } \\ \mathrm{r}=\mathrm{a}=6378136.3 \mathrm{~m}\end{array} \\ 4 & \begin{array}{c}\text { grav disturbance at } \mathrm{r} \\ \text { horizontal gravity }\end{array} & \begin{array}{c}\text { potential coeff } \\ \text { potential coeff }\end{array} & \mathrm{a}=6378136.3 \mathrm{~m}\end{array}$

\section{solve.f}

USAGE:

OPTIONS:

solve n_list -Cfile.coe -Llmax -Anarc [-W -M]

$\mathrm{n} \_$list list of files of normal equations

-C file of output coefficients

-A number of orbital arcs

-L max harmonic degree

-W use weighted constraints [default: no weighted constraint]

-M solve for coefficients of temporal gravity variation. weighted constraint

are automatically added

\section{DESCRIPTION:}

solve.f solves for geopotential coefficients given normal equations from programs norm.f or norm_t2.f.

\section{steric.f}

USAGE:

steric sst.dat -Asstaa.dat -Bsteric.dat

OPTIONS:

sst.dat gridded temerature data at depths

-A output file of temperature anomalies (long., lat., and anomaly)

-B output file of steric anomaly (long., lat., and anomaly)

\section{DESCRIPTION:}

steric.f computes steric anomaly of sea surface due to the thermal expansion, 
based on a 14-layer model of Chen et al. (2000). The expansion coefficients

are from Gill (1982). The gridded temperature data are both in space and time.

syn.f

USAGE:

syn coef_file -Lnmax -Idx/dy -Gfile.grd1 -Ttype [-Mgm -Aae -Dr -R -B]

OPTIONS:

coef_file file of harmonic coefficients

-L max degree of spherical harmonic expansion

-G output file in .grd1 format (see type)

-I grid interval (in degrees) along longitude and latitude

-T type of value to compute [default $=0$ ]

$0=$ geoid undulation, $1=$ gravity anomaly, $2=$ arbitrary function

-M product of Newtonian constant and the mass of the earth

[default: $3986004.415 \mathrm{E}+8 \mathrm{~m} * * 3 / \mathrm{s} * * 2$ ]

-A a scaling factor associated with Cnm and Snm coefficients (See Note 3)

$-\mathrm{D}$ radius (in meter) of sphere on which the expansion is made

[default: $\mathrm{r}=\mathrm{ae}=6378136.3 \mathrm{~m}$ ]

-R remove reference geopotential coefficient of the GRS80 ellipsoid

[default: do not remove]

-B coefficient file is a binary file [deafult: ascii]

\section{varmod.f}

USAGE:

varmod field.var -Lnmax

OPTIONS:

field.var file degree and degree variance of a field

nmax max degree under consideration

\section{DESCRIPTION:}

varmod.f least-squares fits the averaged degree variances (sigma_n/ $(2 n+1)$ )

by the model: alpha $\mathrm{x}$ degree**(-beta). The output contains alpha, beta and a list of

degrees and modeled degree variances. 


\section{References}

Balmino, G., 1994. Orbit choice and the theory of radial orbit error for altimetry, in: Satellite Altimetry in Geodesy and Oceanography. Sansò F and Rummel R (eds) Lecture Notes in earth sciences, Vol. 50, Springer, Berlin, pp. 244-317.

Balmino, G., F. Perosanz, R. Rummel, N. Sneeuw, H. Sünkel, and P. Woodworth, 1998. European views on dedicated gravity field missions: GRACE and GOCE, ESA Rep. ESDMAG-REP-CON-001.

Bertiger, W.I., et al., 1994. GPS precise tracking of TOPEX/POSEIDON: results and implications, J. Geophys. Res., 99, 24449-24464.

Chao, B.F., 1993. The geoid and earth rotation, in: Geoid and Its Geophysical Interpretations. Vanicék P and Christou NT (eds) CRC Press, Boca Raton.

Chao, B.F., E. C. Pavlis, C. Hwang, C.C. Liu, C. K. Shum, C. L. Tseng, and M. Yang, 2000. COSMIC: geodetic applications in improving earth's gravity model, Terr., Atm. Ocean. Sci., in press.

Chen, J.L., C.K. Shum, C.R. Wilson, D.P. Chambers, and B.D. Tapley, 2000. Seasonal sea level change from TOPEX/POSEIDON observation and thermal expansion, J. Geod., 73, 638-647.

Colombo, O., 1981. Numerical Method for Harmonic Analysis on the Sphere. Rep. No. 310, Dept. of Geod. Sci., Ohio State Univ., Columbus.

Colombo, O., 1984. Altimetry, Orbits and Tides, NASA TM 86180, Greenbelt, Maryland.

Engelis, T., 1987. Radial Orbit Error Reduction and Sea Surface Topography Determination Using Satellite Altimetry, Dept. of Geodetic Science and Surveying, Rep. No. 377, The Ohio State University, Columbus.

Fu, L. -L., E. J. Christensen, C. A. Yamarone Jr, M. Lefebvre, Y. Menard, M. Dorrer, and P. Escudier, 1994. TOPEX/POSEIDON mission overview, J. Geophys. Res., 99, 24369-24382.

Gill, A. E., 1982. Atmosphere-Ocean Dynamics, Academic Press, New York.

Heiskanen, W.A., and H. Moritz, 1985. Physical Geodesy, reprint, Inst. of Physical Geodesy, TU Graz, Austria.

Hwang, C., 1995. Orthonormal function approach for Geosat determination of sea surface topography., Mar. Geod., 18, 245-271.

Hwang, C. and M-J. Lin, 1998. Fast integration of low orbiter' trajectory perturbed by the earth's non-sphericity, J. Geod., 72, 578-585.

Jekeli, C., and R. Garcia, 1996. Direct determination of Vehicle acceleration using GPS phase observables, ION 52 Annual Meeting, Cambridge, MA, June 19-21.

Kaula, W.M., 1966. Theory of Satellite Geodesy, Blaisdell Pub. Co., London.

Krogh, F.T., 1974. Changing the stepsize in the integration of differential equations using modified divided differences, in: Proc. of the Conference on the Numerical Solution of Ordinary Differential Equations. Dold A and Eckmann B (eds) Lecture Notes in Mathematics, Vol. 362, Springer-Verlag, Berlin, pp. 22- 71.

Kuo, Y.H., and L. C. Lee, 1999. A constellation of microsatellites promises to help in a range of geoscience research, EOS trans., AGU, 80 (40), p. 467.

Lemoine, F. G., et al., 1998. The Development of the Joint NASA GSFC and the National Imagery and Mapping Agency (NIMA) Geopotential Model EGM96, NASA/TP-1998206861, Greenbelt, Maryland.

Mesk, A., 1984. Digital Filtering: Applications in Geophysical Exploration for Oil. Pitman Pub. Ltd., London. 
Paul, M. K., 1978. Recurrence relations for integrals of associated Legendre functions, Bull. Geod., 52, 177-190.

Pavlis, D. E., et al., 1996. GEODYN Operational Manual, 5 volumes, Hughes/STX Corp., Greenbelt, Maryland.

Rapp, R.H., 1989. Combination of satellite, altimetric and terrestrial gravity data, in: Theory of Satellite of Geodesy and Gravity Field Determination. Sansò F and Rummel R (eds) Lecture Notes in earth sciences, Vol. 25, Springer, Berlin, pp. 261-284.

Reigber, C., 1989. Gravity recovery from satellite tracking data, in: Theory of Satellite of Geodesy and Gravity Field Determination. Sansò F and Rummel R (eds) Lecture Notes in earth sciences, Vol. 25, Springer, Berlin, pp. 197-234.

Rim, H.J., G.W Davis, and B.E. Schutz, 1996. Dynamic orbit determination for the EOS laser satellite (EOS ALT/GLAS) using GPS measurements, J. Astron. Sci., 44, 409-424.

Rosborough, G.W., and B.D. Tapley, 1987. Radial, Transverse and normal satellite position perturbations due to the geopotential, Cel. Mech., 40, 409-421.

Schrama, E.J.O., 1991. Gravity field error analysis: Applications of Global Positioning System receivers and gradiometers on low orbiting platforms, F. Geophys. Res., 96 (B12), 2004120051.

Wahr, J., M. Molenaar, and F. Bryan, 1998. Time variability of the earth's gravity field: Hydrological and oceanic effects and their possible detection using GRACE, JGR, 103, 30205-30229.

Wagner, C.A., 1985. Radial Variations of a Satellite Orbit Due to Gravitational Errors: Implications for Satellite Altimetry, J. Geophys. Res., 90, 3027-3036. 